\title{
Structure detection in the D1 CFHTLS deep field using accurate photometric redshifts: a benchmark ${ }^{\star}$
}

\author{
A. Mazure ${ }^{1}$, C. Adami ${ }^{1}$, M. Pierre ${ }^{2}$, O. Le Fèvre ${ }^{1}$, S. Arnouts ${ }^{1}$, P. A. Duc ${ }^{2}$, O. Ilbert ${ }^{3}$, V. LeBrun ${ }^{1}$, B. Meneux ${ }^{1,4,5}$, \\ F. Pacaud $^{2}$, J. Surdej $^{3}$, and I. Valtchanov 6 \\ 1 LAM, Traverse du siphon, 13012 Marseille, France \\ e-mail: christophe.adami@oamp.fr \\ 2 DAPNIA/SAp, CEA Saclay, 91191 Gif-sur-Yvette, France \\ 3 Institut d'Astrophysique et de Géophysique, Université de Liège, 17 Allée du 6 Août, B5C, 4000 Sart Tilman, Belgique \\ 4 INAF - IASF, via Bassini 15, 20133 Milano, Italy \\ 5 INAF, Osservatorio Astronomico di Brera, via Bianchi 46, 23807 Merate (LC), Italy \\ 6 Herschel Science Centre, ESA, European Space Astronomy Centre (ESAC), Villafranca del Castillo, PO Box 50727, Madrid 28080, \\ Spain
}

Received 11 September 2006 / Accepted 13 February 2007

\begin{abstract}
Aims. We investigate structures in the D1 CFHTLS deep field to test the method that will be applied to generate homogeneous samples of clusters and groups of galaxies in order to constrain the cosmology and detailed physics of groups and clusters.

Methods. An adaptive kernel technique was applied to galaxy catalogues. This technique needs none of the usual a-priori assumptions (luminosity function, density profile, colour of galaxies) made with other methods. Its main drawback (decrease in efficiency with increasing background) is overcome by the use of narrow slices in photometric redshift space. There are two main concerns in structure detection. One is false detection and the second, the evaluation of the selection function in particular if one wants complete samples. We deal with the first concern using random distributions. For the second, comparison with detailed simulations is foreseen but we used a pragmatic approach by comparing our results to GalICS simulations to check that our detection number is not totally at odds with cosmological simulations. We used the XMM-LSS survey and secured VVDS redshifts up to $z \sim 1$ to check individual detections.

Results. We show that our detection method is basically able to recover 100\% of the C1 XMM-LSS X-ray detections (in the regions in common) in the correct redshift range plus several other candidates. Moreover, when spectroscopic data are available, we confirm our detections, even those without X-ray data.
\end{abstract}

Key words. galaxies: clusters: general

\section{Introduction}

Considering groups and clusters of galaxies as cosmological probes, two questions are still being asked: when these structures were formed and could they constrain model universes if we consistently count them with redshift and mass. Obviously, both questions point out the need for complete samples of clusters and groups to answer them. Moreover, once these samples are available, the detailed physics of galaxy groups and clusters can be studied. With the CFHTLS survey (Canada-France Hawaii Telescope Legacy Survey, see http://www. cfht . hawaii.edu/Science/CFHLS) this could be done up to $z \geq 1$ extending e.g. recent GEMS study (Forbes et al. 2006) on nearby groups.

Physically, groups and clusters are deep gravitational potential wells containing dark matter, hot gas, and galaxies. Each of these components could then be used to detect their parent host.

a) Dark matter (DM hereafter). This phase is usually sampled via lensing studies (e.g. Gavazzi \& Soucail 2006, on the same CFHTLS fields and references therein), since DM

$\star$ Table A.1 is only available in electronic form at http://www . aanda.org traces the mass directly. However, lensing detection is efficient up to $z$ systems that are not too high (Hamana et al. 2003), due to projection effects and lack of sensitivity to small groups. Moreover, due to the complex mass distribution of DM haloes and the spoiling influence of intrinsic alignments of galaxies, lensing-selected clusters could not really be mass-selected (e.g. Tang \& Fan 2005).

b) Hot gas. This can be achieved in two ways. First, X-rays (e.g. Pierre et al. 2006, and references therein) should probe gravitational wells without projection effects, but they are contaminated by line-of-sight stars or active galaxies. Limited exposure times also become problematic for faint remote clusters. Second, the Sunyaez-Zeldovich (SZ hereafter) technique works at any redshift, but in practice there are limitations for distant objects due to the lack of spatial resolution.

c) Galaxies. First, for this component, spectroscopic redshift surveys are in principle the optimal tool since they directly probe the dynamics of the systems. Most of the time, however, the spatial sampling is partial and not homogeneous. Moreover, high-redshift clusters are not probed well due to slits/fibers overlap problems. Second, pure photometric catalogues can be used, searching for galaxy overdensities over the sky. This method generally provides a homogeneous spatial coverage, but the contrast of 
structures decreases rapidly with redshift with respect to the total background. Improvements are possible by using matching filter techniques (Postman et al 1996 for the seminal work; Olsen et al. 2007 on the CFHTLS fields) and/or selecting galaxies on a colour-basis (using the red sequence in the colour-magnitude relation e.g. Gladders \& Yee 2000, 2005) but this method possibly introduces a bias in the sense that it only searches for clusters exhibiting such a relation. Moreover, even if the colour-magnitude relation already seems to be in place up to $z \sim 1.5$ (e.g. Cucciati et al. 2006 for a complete discussion), the colour-magnitude distribution dependence upon environment also varies with redshift. As a result, one would ideally have to adapt the cluster search using the colour-magnitude relation not only to the redshift but also to the local density. This kind of improvement was also made more general in Miller et al. (2005), identifying clusters of galaxies as overdensities in a seven-dimensional position and colour space to minimize the projection effects.

We chose a similar way to solve the problem of this lack of contrast in using photometric redshifts to define redshift slices. But, up to now, the accuracy of such redshifts was moderate and significant numbers of totally wrong redshift estimates were still present. However, impressive improvements have been recently made due to training of spectral templates and calibration with very good and large spectroscopic samples over a wide range of redshifts and down to similar magnitude depth compared to the photometric catalogues. We therefore used such improved photometric redshifts (Ilbert et al. 2006) in order to define redshift slices that allowed us to increase the contrast when computing galaxy density maps. This results in a homogeneous sample (besides masked photometric areas) only limited by the photometric catalogue depth.

We took advantage of both excellent multi-wavelength photometry from CFHTLS and very large samples of spectra (VVDS survey: VIMOS VLT Deep Survey, Le Fèvre et al. 2005) to define these very good photometric redshifts in the CFHTLS D1 field, so we exploit them here to search for structures in that field. We postpone the use of counts of structures to constrain cosmological parameters to another paper in which all the CFHTLS fields will be analysed. Indeed, only a few systems are expected in less than 1 square degree. Instead, we stress how the method appears efficient. In particular, a close comparison to the $\mathrm{X}$-ray detections done in the framework of the XMM-LSS survey (e.g. Pierre et al. 2006) shows very good agreement when taking biases in both methods into account and we will also explain this comparison using secured VVDS redshifts.

Section 2 is about data and methods. Section 3 describes the structure detection and the resulting reliability. Finally, Sect. 4 is the conclusion. We use the following cosmological parameters: $H_{0}=67 \mathrm{~km} \mathrm{~s}^{-1} \mathrm{Mpc}^{-1}, \Omega_{\Lambda}=0.67$ and $\Omega_{\mathrm{m}}=0.33$ in order to be coherent with the GalICS simulations.

\section{Data and method}

\subsection{CFHTLS photometric data and photometric redshifts}

The catalogues (publicly available and fully described at http: //terapix .iap. fr) used for the detection and the characterisation of the structures have been obtained within the framework of the CFHT Legacy Survey (i.e. MEGACAM $u^{*}$, $g^{\prime}, r^{\prime}, i^{\prime}, z^{\prime}$ data) for the so-called D1 deep field. We used the $i^{\prime}$ band to detect our structures (see below).

For the photometric redshift calculations, photometry $(B V R I)$ obtained in the framework of the VVDS survey
(Le Fèvre et al. 2005), as well as Spitzer data, are also used (see Ilbert et al. 2006, for details). In a few words, photometric redshifts were obtained by adjusting spectral energy distribution of galaxy templates, which are iteratively modified in terms of flux zero points and continua shapes using a set of high-quality spectroscopic redshifts issued from the VVDS (see Ilbert et al. 2006). A very good accuracy was obtained between $z=0.2$ and $z=1.2$ and for $i^{\prime}=24.5$ (the present limit of our sample, see below) as described in Ilbert et al. (2006): in a photometric/spectroscopic redshift plot, the standard deviation is 0.04 in redshift.

\subsection{The VVDS spectroscopic data and XMM data}

Once the structures are identified with their galaxy content (see following sections), we look for spectroscopic data in the VVDS catalogue both to confirm our detections and to give an estimate of the velocity dispersion if possible. However, the VVDS does not cover the entire D1 field and is characterised by an inhomogeneous sampling rate; and it avoids peculiar masked regions (distinct from the CFHTLS D1 masked regions, however), so several systems have only a few spectroscopic measurements or not at all.

On the other hand, the XMM-LSS (Pierre et al. 2006) provides for the CFHTLS D1 covered area, a catalogue of candidate structures classified in several classes $(\mathrm{C} 1, \mathrm{C} 2, \mathrm{C} 3)$ with confirmed spectroscopic redshifts (independently from VVDS redshifts). Class 1 (C1) is defined as sources with no contamination by point sources interpreted as extended ones. Class 2 (C2) corresponds to a contamination of $50 \%$ and class 3 (C3) to highly contaminated ones (see Pierre et al. 2006, for more details). We also used the identifications by Willis et al. (2005) and Andreon et al. (2005) of specific systems with the same X-ray data.

First of all, Fig. 1 shows the CFHTLS D1 masked regions due to bright stars and the CCD defects of the optical data. Masked regions are represented by the spurious objects that were detected inside. Rings made by agglomerated points with empty centres are for example due to stars that shield part of the sky (i.e. empty centres) and contamine the immediate vicinity with their diffuse light (e.g. rings of points).

It allowed us to check whether an XMM source is found in a masked region and then if any optical detection could be spoiled by this masking (e.g. XLSSC 029 at the upper left edge of the D1 Megacam field). Second, we give the various XMMLSS fields in Fig. 2 along with their corresponding associated exposure times (which can vary by a factor of 2) and the $\mathrm{C} 1,2$, and 3 cluster detections. The part of the field at both small right ascension and declination with no XMM observations at all is called the "Absence zone" in the following.

\subsection{Galaxy density maps}

The method is based on the simple detection of contrasts in numerical density maps of galaxies computed using $i^{\prime}$ band data. But, to eliminate fore and background contaminations as much as possible, these density maps are built in redshift (distance) slices. The technique used to compute the galaxy density maps is the well-known adaptive kernel method; see e.g. Dressler \& Shectman (1988), Beers et al. (1991) for seminal works and also Biviano et al. (1996) for a detailed application and discussion of significance. It has the advantage over wavelets (e.g. Escalera et al. 1992; Slezak et al. 1994) of not needing reconstruction of the structures using the whole range of scales and is less affected by edge effects. Edge effects, as well as mask effects (masks due 


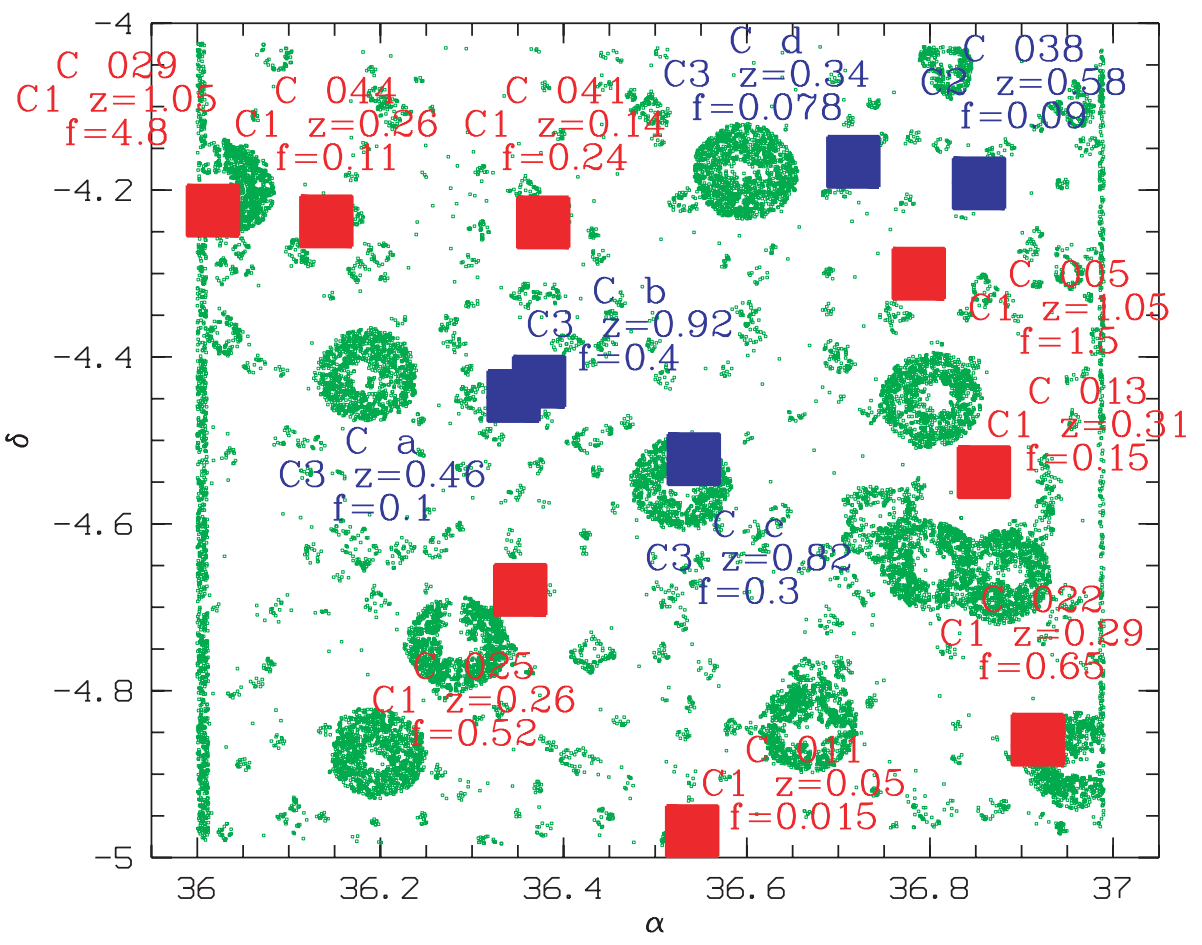

Fig. 1. Masked regions following the CFHTLS recipe on the D1 field with the XMM detections superimposed. Red filled squares are the C1 XMM-LSS clusters and blue ones are the C2 and C3. We also give the name of the XMM-LSS cluster (see Pierre et al. 2006), as well as its redshift and bolometric X-ray luminosity in $10^{44} \mathrm{erg} / \mathrm{s}$ within $R_{500} . \alpha$ and $\delta$ are given in decimal degrees.

for example to the presence of bright stars in the field), are taken into account neither by mirroring the data nor by adding randomly distributed points. We compared our detections with the map of masks and eyeballed if there was any unfortunate coincidence. Systems where the centre of the contours was in such a masked region were flagged by an (M) in the lists of candidates, if not already associated with an X-ray structure.

In this testing approach, we prefer to deal with crude artefacts rather than smoothing them in order to estimate their effects when compared to totally different detection methods. In an application aimed at counting groups and clusters to constrain cosmology, it turns out that the best way would be to, a posteriori, totally exclude masked regions as done e.g. when using lensing techniques. For the edge effects, comparison of our detections with real structures detected in X-ray will indicate later that the effect does not seem to be very strong. Again, when counting structures, this should be taken into account by removing adequate border zones. Only foreseeing a comparison with simulations, including fake structures where completeness in terms of richness or mass is well-controlled, will allow quantitative estimates of these effects.

We thus defined overlapping slices with a width of 0.1 in photometric redshift all along the line of sight. An overlap of 0.05 was chosen, as real structures are often expected to show up in adjacent slices. The D1 field is $0.8 \mathrm{deg}^{2}$ (taking into account the masked area rejection) and we define a grid of $200 \times 200$ pixels on it. The pixel size $(\sim 0.3 \mathrm{arcmin})$ corresponds to $\sim 80 / 120 / 160 \mathrm{kpc}$ at $z=0.25 / 0.5 / 1$ and is kept fixed with redshift.

To establish statistical significance, we used a bootstrap technique both on the real data and simulations. For each new realisation of a given galaxy distribution obtained by the bootstrap technique, we built the corresponding density map, and clustered points on small scales stay clustered but are spread out. Points

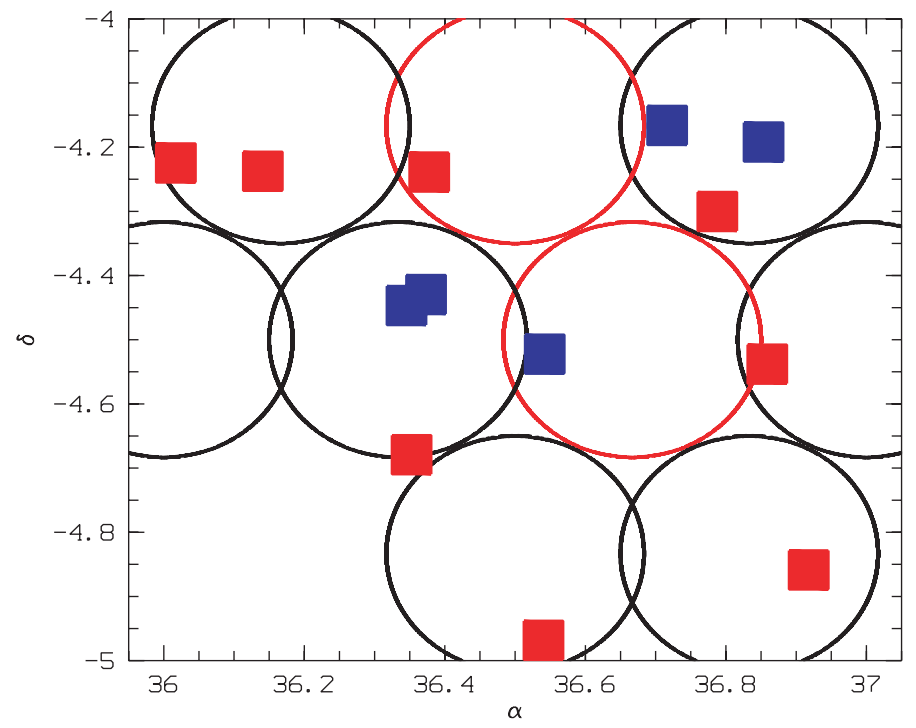

Fig. 2. Regions observed by XMM with the corresponding $\mathrm{C} 1, \mathrm{C} 2$, and C3 detections as in Fig. 1 (see Pierre et al. 2006). The red circles have a shorter X-ray exposure time. Red filled squares are the C1 XMM-LSS clusters and blue ones are the $\mathrm{C} 2$ and $\mathrm{C} 3$.

that are unclustered or clustered on larger scales are also spread out. Then, taking the mean of several density maps leads to erase fluctuations and flattens the mean background, keeping clustering present. This flattening added to the removal of distant (or nearby) clustering due to the use of narrow slices allows using random distributions to evaluate false detections, at least when using high-value thresholds. In practice, a mean bootstrapped map of the galaxy distribution within a given zphot slice is obtained using 1000 bootstrap resamplings (see e.g. Biviano et al. 1996, for a complete description). 


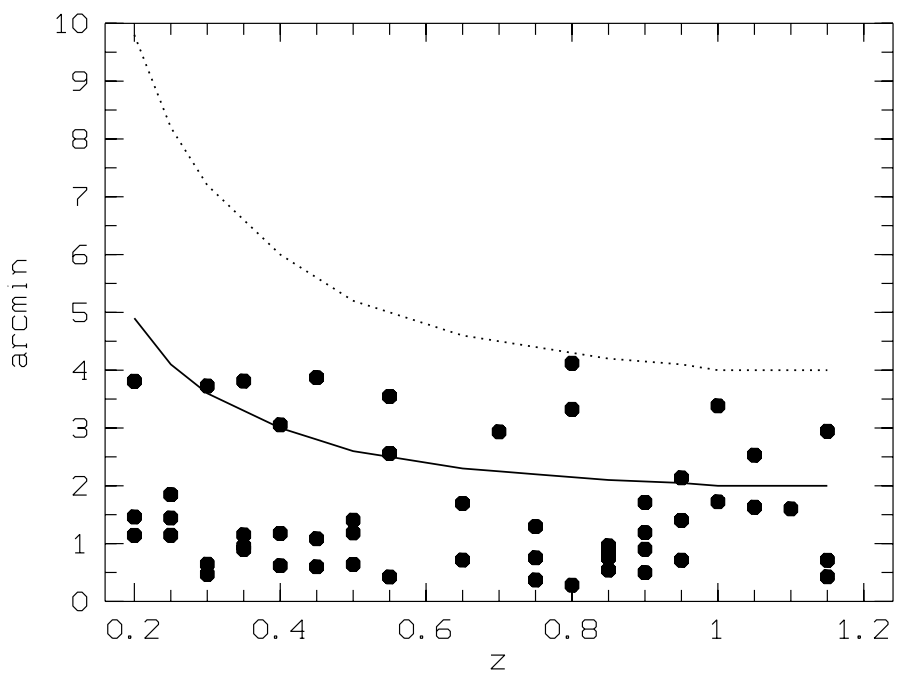

Fig. 3. Identification distance in arcmin between two detections (in successive redshift slices) that are assumed to be the same structure as a function of redshift. Solid and dashed lines are (in arcmin) the 1 and $2 \mathrm{Mpc}$ values as a function of redshift.

Table 1. Number of peaks detected per square degree in 100 random fields with respect to a given threshold.

\begin{tabular}{cccc}
\hline \hline Threshold & 5000 points & 8000 points & 11000 points \\
\hline$\geq 3-\sigma$ & $0.7 \pm 0.8$ & $0.6 \pm 0.6$ & $1.6 \pm 0.9$ \\
$\geq 4-\sigma$ & $0.1 \pm 0.2$ & $0.2 \pm 0.4$ & $0.6 \pm 0.6$ \\
\hline
\end{tabular}

Table 2. Number of peaks detected per square degree in 50 GalICS fields (averaged over the 50 available fields) and in real D1 fields (rescaled to $1 \mathrm{deg}^{2}$ ) with respect to redshift and to a given threshold. We only give the non-overlapping redshift slices.

\begin{tabular}{ccccc}
\hline \hline$z$ & $\geq 3 \sigma \mathrm{Gal}$ & $\geq 4 \sigma \mathrm{Gal}$ & $\geq 3 \sigma \mathrm{D} 1$ & $\geq 4 \sigma \mathrm{D} 1$ \\
\hline $0.2-0.3$ & $7.0 \pm 0.8$ & $6.2 \pm 0.8$ & 7.5 & 5.0 \\
$0.3-0.4$ & $8.0 \pm 0.8$ & $6.8 \pm 0.8$ & 5.0 & 3.75 \\
$0.4-0.5$ & $8.1 \pm 0.9$ & $6.8 \pm 1.0$ & 5.0 & 1.25 \\
$0.5-0.6$ & $8.3 \pm 0.9$ & $6.6 \pm 1.0$ & 7.5 & 3.75 \\
$0.6-0.7$ & $8.0 \pm 0.9$ & $6.0 \pm 1.1$ & 2.5 & 1.25 \\
$0.7-0.8$ & $7.6 \pm 1.1$ & $5.4 \pm 0.7$ & 5.0 & 1.25 \\
$0.8-0.9$ & $6.9 \pm 0.7$ & $4.7 \pm 0.8$ & 6.25 & 2.5 \\
$0.9-1.0$ & $6.3 \pm 0.6$ & $4.3 \pm 0.6$ & 6.25 & 2.5 \\
$1.0-1.1$ & $5.6 \pm 0.6$ & $3.6 \pm 1.0$ & 3.75 & 2.5 \\
$1.1-1.2$ & $4.9 \pm 0.6$ & $3.0 \pm 0.5$ & 5.0 & 2.5 \\
$1.2-1.3$ & $5.4 \pm 0.5$ & $3.2 \pm 0.6$ & 6.25 & 5.0 \\
\hline
\end{tabular}

\section{Application to real data and reliability}

The present analysis was performed with the $i^{\prime}$ band CFHLS catalogue, down to the magnitude limit $i^{\prime}=24.5$, which encompasses the typical $i^{*}$ of the luminosity function at $z=1$ (e.g. Adami et al. 2005) and which ensures a good compromise between using the reddest possible band and the best quality photometric data.

We applied the technique to overlapping slices with central redshifts between 0.2 and 1.2 (where the quality of photometric redshifts is optimal). We added the slice $0.10-0.20$ to check if the nearby $\mathrm{C} 1 / 0.041$ is detected by our method. We also give the tentative detections up to $z=1.5$ in the appendix, but these results are still uncertain due to the decrease in the photometric redshift accuracy after $z=1.2$. This is also why the last redshift slice in the appendix was taken larger (width of 0.15 in redshift) than the other ones.

Once the mean image is computed (from 1000 realisations obtained by bootstrapping the actual data), the peaks are detected. This detection uses the classical image analysis with Sextractor (Bertin \& Arnouts 1996) where the internal parameters are adapted to the pixel size with at least 2 pixels above the chosen threshold (3 or 4). As in the usual image analyses, structures are detected with respect to their background (estimated globally in Sextractor), which defines a threshold (peak density over background density). Positions and best ellipse fitting (orientation and axes ratio) are derived by adopting $1 \mathrm{Mpc}$ as the size of the semi-major axis of these structures.

A catalogue of structures for each redshift slice was then generated. We also selected individual galaxies potentially belonging to each structure as for all galaxies included in the considered redshift slice and in the $1 \mathrm{Mpc}$ semi-major axis ellipse. For a given structure, these lists still include, however, interloper galaxies that have positions inside the structure ellipse but are the foreground or background galaxies included in the redshift slice. This is due to the photometric redshift uncertainty (see Ilbert et al. 2006).

We avoided the very low redshift slices because, in that case, the number of expected structures is small due to the small solid angle, and the reliability of photometric redshifts is degraded (see Ilbert et al. 2006). We also provide detections only up to $z=1.2$ (tentative detections up to $z=1.5$ are only given in appendix), but we clearly waited for complete comparison with the next generation of simulations to validate and study detections of the most distant candidate clusters in terms of mass. As we used galaxy as tracers of structures, it is important to deal with largescale structure simulations that include a well-controled implementation of galaxies and not only of DM. It is, however, encouraging that the number of detections agree closely with generic GalICS predictions (see below).

Every significant (i.e. in terms of threshold, see below) structure is labelled with an identification in every slice, as well as a general identification. Some structures could show up at almost the same positions in several slices. To identify these multiple detections, we chose to offer a single identification when two detections in adjacent redshift slices had overlapping ellipses on the sky. We show in Fig. 3 the coordinate differences of all multiple detections in successive redshift slices. This figure shows that two successive detections are always closer than $2 \mathrm{Mpc}$ (by definition) and that more than $75 \%$ are closer than $1 \mathrm{Mpc}$. The values are roughly 2 times (or smaller) the usual virial radius, which ensures that, most of the time, we are not merging unrelated structures. The other detections of a given structure are then labelled by the same number but flagged by parentheses in Tables 3 to 5 , which summarise the structures we found.

We note, however, that when a structure (e.g. large-scale structure line-of-sight filaments) is percolating through a large number of redshift slices, the position of the lowest redshift detection can be quite different from the one in the highest redshift slice. We also note that C1-029 from Pierre et al. (2006) is just on the edge of the D1 field of view and is also located in a masked region. It is perhaps identified with cluster 35 (general ID of the tables), but this remains very uncertain.

Finally, since part of the D1 field is covered by VVDS data, we used the spectroscopic information. In every $1 \mathrm{Mpc}$ ellipse, when available, we looked at spectroscopic data in the 0.1 width redshift range to confirm that the local $z$ distribution exhibits any compactness in the velocity space compatible with the presence of possible real structures. We looked at galaxies along the line 
Table 3. Structures detected in redshift slices with central redshift from 0.15 to 0.70 .

\begin{tabular}{|c|c|c|c|c|c|c|c|c|}
\hline Slice & $\overline{\mathrm{ID}}$ & $\alpha$ & $\bar{\delta} \delta$ & Min. thres. & Gen. ID & $\overline{X \text { X-ray ID and } z \text { XMM }}$ & T-X & $\overline{\text { VVDS }}$ \\
\hline \multirow[t]{2}{*}{$0.10-0.20(5868)$} & 0001 & 36.3789 & -4.2424 & 4 & 1 & $\mathrm{C} 1-041 / 0.14$ & $1.3 \mathrm{keV}$ & $\mathrm{Y}$ \\
\hline & 0002 & 36.7981 & -4.1970 & 3 & 2 & & & Y \\
\hline \multirow[t]{4}{*}{$0.15-0.25(7848)$} & 0001 & 36.3746 & -4.6831 & 3 & 3 & C1-025/0.26 & $2.0 \mathrm{keV}$ & $\mathrm{Y}$ \\
\hline & 0002 & 36.5767 & -4.0699 & 3 & (4) & $\mathrm{S}$ & & $\mathrm{N}$ \\
\hline & 0003 & 36.6240 & -4.2523 & 3 & 5 & $\mathrm{~S}$ & & Y \\
\hline & 0004 & 36.8215 & -4.5465 & 3 & (6) & $\mathrm{S}$ & & Y \\
\hline \multirow[t]{6}{*}{$0.20-0.30(9252)$} & 0001 & 36.8946 & -4.8679 & 4 & 7 & C1-022/0.29 & $1.7 \mathrm{keV}$ & $\mathrm{N}$ \\
\hline & 0002 & 36.2451 & -4.8901 & 3 & 8 & $\mathrm{~A} / \mathrm{M}$ & & $\mathrm{N}$ \\
\hline & 0003 & 36.1364 & -4.2134 & 4 & 9 & C1-044/0.26 & $1.3 \mathrm{keV}$ & \\
\hline & 0004 & 36.6840 & -4.2315 & 3 & (5) & & & $\mathrm{Y}$ \\
\hline & 0005 & 36.8435 & -4.5570 & 4 & (6) & & & Y \\
\hline & 0006 & 36.5928 & -4.0801 & 4 & (4) & $\mathrm{S}$ & & $\mathrm{N}$ \\
\hline \multirow[t]{6}{*}{$0.25-0.35(10570)$} & 0001 & 36.3166 & -4.7515 & 3 & 10 & MA & & $\mathrm{Y}$ \\
\hline & 0002 & 36.9117 & -4.8594 & 4 & (7) & & & $\mathrm{N}$ \\
\hline & 0003 & 36.6381 & -4.8759 & 4 & 11 & M & & $\mathrm{N}$ \\
\hline & 0004 & 36.8416 & -4.5810 & 4 & 6 & C1-013/0.31 & $1.0 \mathrm{keV}$ & Y \\
\hline & 0005 & 36.6121 & -4.0741 & 3 & (4) & & & $\mathrm{N}$ \\
\hline & 0006 & 36.6104 & -4.5286 & 3 & 12 & $\mathrm{~S}$ & & Y \\
\hline \multirow[t]{4}{*}{$0.30-0.40(9973)$} & 0001 & 36.2974 & -4.7511 & 3 & (10) & $\mathrm{A} / \mathrm{M}$ & & $\mathrm{Y}$ \\
\hline & 0002 & 36.6445 & -4.8804 & 4 & (11) & M & & $\mathrm{N}$ \\
\hline & 0003 & 36.8090 & -4.6339 & 4 & (6) & $\mathrm{G} / \mathrm{M}$ & & $\mathrm{Y}$ \\
\hline & 0004 & 36.6215 & -4.0689 & 4 & 4 & XLSS014/W05/0.34 & & $\mathrm{N}$ \\
\hline \multirow[t]{4}{*}{$0.35-0.45(8248)$} & 0001 & 36.1413 & -4.8965 & 4 & 13 & $\mathrm{~A} / \mathrm{M}$ & & $\mathrm{N}$ \\
\hline & 0002 & 36.6542 & -4.9432 & 4 & (11) & $\mathrm{G}$ & & $\mathrm{N}$ \\
\hline & 0003 & 36.7937 & -4.6294 & 3 & (6) & $\mathrm{G} / \mathrm{M} / \mathrm{S}$ & & Y \\
\hline & 0004 & 36.6156 & -4.0665 & 3 & (4) & S & & $\mathrm{N}$ \\
\hline \multirow[t]{4}{*}{$0.40-0.50(8302)$} & 0001 & 36.1201 & -4.8502 & 4 & (13) & $\overline{\mathrm{A}}$ & & $\overline{\mathrm{N}}$ \\
\hline & 0002 & 36.6735 & -4.9467 & 4 & (11) & G & & $\mathrm{N}$ \\
\hline & 0003 & 36.0884 & -4.0633 & 3 & (14) & & & $\mathrm{N}$ \\
\hline & 0004 & 36.6258 & -4.0680 & 3 & (4) & $\mathrm{S}$ & & $\mathrm{N}$ \\
\hline \multirow[t]{6}{*}{$0.45-0.55(9216)$} & 0001 & 36.1152 & -4.8328 & 4 & (13) & A & & $\overline{\mathrm{N}}$ \\
\hline & 0002 & 36.6799 & -4.0610 & 3 & (4) & G & & $\mathrm{N}$ \\
\hline & 0003 & 36.0798 & -4.0684 & 4 & 14 & & & $\mathrm{~N}$ \\
\hline & 0004 & 36.3842 & -4.2726 & 4 & 15 & $\mathrm{~S}$ & & Y \\
\hline & 0005 & 36.8975 & -4.3768 & 3 & 16 & & & Y \\
\hline & 0006 & 36.8481 & -4.6202 & 3 & 17 & $\mathrm{G} / \mathrm{M}$ & & Y \\
\hline \multirow[t]{6}{*}{$0.50-0.60(10201)$} & 0001 & 36.1202 & -4.8557 & 4 & (13) & $\mathrm{A}$ & & $\mathrm{N}$ \\
\hline & 0002 & 36.6990 & -4.0559 & 3 & (4) & & & $\mathrm{N}$ \\
\hline & 0003 & 36.0758 & -4.1999 & 4 & 18 & M & & $\mathrm{N}$ \\
\hline & 0004 & 36.3762 & -4.2655 & 4 & (15) & $\mathrm{S}$ & & Y \\
\hline & 0005 & 36.8334 & -4.4982 & 3 & (19) & M & & Y \\
\hline & 0006 & 36.2840 & -4.7423 & 3 & 20 & $\mathrm{~A} / \mathrm{M}$ & & $\mathrm{N}$ \\
\hline \multirow[t]{4}{*}{$0.55-0.65$ (10845) } & 0001 & 36.1366 & -4.8951 & 3 & (13) & $\mathrm{A}$ & & $\mathrm{N}$ \\
\hline & 0002 & 36.3825 & -4.2687 & 3 & (15) & $\mathrm{S}$ & & Y \\
\hline & 0003 & 36.4645 & -4.4997 & 3 & 21 & & & Y \\
\hline & 0004 & 36.8646 & -4.5484 & 4 & 19 & & & Y \\
\hline \multirow[t]{2}{*}{$0.60-0.70(10224)$} & 0001 & 36.6686 & -4.5096 & 4 & 22 & $\bar{S}$ & & $\bar{Y}$ \\
\hline & 0002 & 36.2364 & -4.2232 & 3 & (23) & & & $\mathrm{N}$ \\
\hline \multirow[t]{5}{*}{$0.65-0.75(9243)$} & 0001 & 36.0853 & -4.7942 & 3 & 24 & $\mathrm{~A}$ & & $\mathrm{~N}$ \\
\hline & 0002 & 36.4815 & -4.0820 & 3 & 25 & $\mathrm{M} / \mathrm{S}$ & & $\mathrm{N}$ \\
\hline & 0003 & 36.7546 & -4.0752 & 3 & 26 & & & $\mathrm{~N}$ \\
\hline & 0004 & 36.2089 & -4.2167 & 4 & 23 & & & $\mathrm{~N}$ \\
\hline & 0005 & 36.6585 & -4.5160 & 4 & (22) & $\mathrm{S}$ & & Y \\
\hline
\end{tabular}

Structures detected along with their redshift slice (and the total number of galaxies inside the slice), structure ID, coordinates, minimum detection level, general identification, X-ray association or a potential explanation of the absence of X-ray detection (centre of the structure located in A: the Absence zone, G: a Gap between two X-ray pointings, M: a region strongly affected by masked CFHTLS areas, S: a single XMM field where the exposure time was Short, E: a region with an X-ray detection very close to the CFHTLS D1 field Edge), X-ray temperature when available, and VVDS available redshifts. Some structures show up at almost the same positions in several slices: the secondary detections are then labelled by the same number but flagged by parentheses (the unflagged number is the detection made with the highest signal-to-noise). W05 refers to Willis et al. (2005) and A05 to Andreon et al. (2005).

of sight in the considered slice separated by gaps of less than 0.0026 , so as to use the same gap as in Adami et al. (2005) on similar spectroscopic data. These gaps were adapted to redshift using the $(1+z)$ dependence.

\subsection{False detection-rate evaluation}

We deal with false detections as follows. We generate 100 independent slices with 5000, 8000, and 11000 randomly distributed 
Table 4. Structures detected in redshift slices with central redshift from 0.75 to 1.05 .

\begin{tabular}{|c|c|c|c|c|c|c|c|c|}
\hline Slice & ID & $\alpha$ & $\delta$ & Threshold in $\sigma$ & Gen. ID & $\overline{X \text {-ray ID and } z \text { XMM }}$ & T-X & $\overline{\text { VVDS }}$ \\
\hline \multirow{4}{*}{$0.70-0.80(9585)$} & 0001 & 36.9250 & -4.9052 & 3 & 27 & $\mathrm{M}$ & & $\mathrm{N}$ \\
\hline & 0002 & 36.1835 & -4.1749 & 4 & (23) & & & $\mathrm{N}$ \\
\hline & 0003 & 36.8791 & -4.2025 & 3 & 28 & & & Y \\
\hline & 0004 & 36.4924 & -4.4782 & 3 & (29) & & & Y \\
\hline \multirow[t]{6}{*}{$0.75-0.85(9741)$} & 0001 & 36.0853 & -4.8695 & 3 & (30) & $\bar{A}$ & & $\mathrm{~N}$ \\
\hline & 0002 & 36.7614 & -4.0793 & 3 & 31 & & & $\mathrm{~N}$ \\
\hline & 0003 & 36.1627 & -4.1807 & 4 & (23) & & & $\mathrm{N}$ \\
\hline & 0004 & 36.8789 & -4.2087 & 3 & (28) & & & $\mathrm{Y}$ \\
\hline & 0005 & 36.7091 & -4.2481 & 3 & 32 & & & $\mathrm{~N}$ \\
\hline & 0006 & 36.5030 & -4.4714 & 3 & 29 & $\mathrm{C} 3-\mathrm{c} / 0.82 ?$ & & $\mathrm{Y}$ \\
\hline \multirow[t]{5}{*}{$0.80-0.90(11004)$} & 0001 & 36.0856 & -4.8648 & 4 & 30 & $\mathrm{~A}$ & & $\bar{N}$ \\
\hline & 0002 & 36.8458 & -4.1485 & 3 & (28) & & & Y \\
\hline & 0003 & 36.3781 & -4.1865 & 3 & 33 & $\mathrm{~S}$ & & $\mathrm{~N}$ \\
\hline & 0004 & 36.2113 & -4.2073 & 3 & (23) & & & $\mathrm{N}$ \\
\hline & 0005 & 36.4003 & -4.4181 & 4 & (34) & & & $\mathrm{Y}$ \\
\hline \multirow[t]{5}{*}{$0.85-0.95$ (12019) } & 0001 & 36.0864 & -4.8774 & 3 & (30) & $\mathrm{A}$ & & $\overline{\mathrm{N}}$ \\
\hline & 0002 & 36.0587 & -4.2291 & 3 & (35) & M & & $\mathrm{N}$ \\
\hline & 0003 & 36.3895 & -4.1940 & 4 & (33) & $S$ & & $\mathrm{~N}$ \\
\hline & 0004 & 36.2141 & -4.2231 & 3 & (23) & & & $\mathrm{N}$ \\
\hline & 0005 & 36.3925 & -4.4135 & 4 & 34 & XLSSJ022534.2/A05/0.92 C3-b/0.92 & & $\mathrm{Y}$ \\
\hline \multirow[t]{5}{*}{$0.90-1.00(11658)$} & 0001 & 36.0983 & -4.8933 & 4 & $(30)$ & $\mathrm{A}$ & & $\overline{\mathrm{N}}$ \\
\hline & 0002 & 36.0664 & -4.2379 & 3 & (35) & M & & $\mathrm{N}$ \\
\hline & 0003 & 36.2245 & -4.2340 & 3 & (23) & & & $\mathrm{N}$ \\
\hline & 0004 & 36.2492 & -4.3086 & 4 & 36 & & & $\mathrm{~N}$ \\
\hline & 0005 & 36.3847 & -4.4106 & 3 & (34) & & & $\mathrm{Y}$ \\
\hline \multirow[t]{3}{*}{$0.95-1.05(9998)$} & 0001 & 36.0989 & -4.9052 & 3 & $(30)$ & $\bar{A}$ & & $\overline{\mathrm{N}}$ \\
\hline & 0002 & 36.0872 & -4.2272 & 4 & 35 & $\mathrm{E}$ & & $\mathrm{N}$ \\
\hline & 0003 & 36.2499 & -4.2590 & 4 & (23) & & & $\mathrm{N}$ \\
\hline \multirow[t]{3}{*}{$1.00-1.10(8203)$} & 0001 & 36.7801 & -4.2833 & 3 & 37 & C1-005/1.05 & $3.7 \mathrm{keV}$ & $\mathrm{N}$ \\
\hline & 0002 & 36.0875 & -4.1856 & 4 & (35) & E & & $\mathrm{N}$ \\
\hline & 0003 & 36.2513 & -4.2303 & 4 & (23) & & & $\mathrm{N}$ \\
\hline
\end{tabular}

Table 5. Structures detected in redshift slices with central redshift from 1.10 to 1.20 .

\begin{tabular}{|c|c|c|c|c|c|c|c|c|}
\hline Slice & ID & $\alpha$ & $\bar{\delta}$ & Threshold in $\sigma$ & Gen. ID & X-ray ID and $z$ XMM & T-X & VVDS \\
\hline \multirow[t]{3}{*}{$1.05-1.15(7755)$} & $\overline{0001}$ & 36.2635 & -4.2060 & 3 & $(23)$ & & & $\mathrm{N}$ \\
\hline & 0002 & 36.8640 & -4.2023 & 3 & 38 & & & $\mathrm{~N}$ \\
\hline & 0003 & 36.0848 & -4.2277 & 3 & (35) & $\mathrm{M}$ & & $\mathrm{N}$ \\
\hline \multirow[t]{4}{*}{$1.10-1.20(7612)$} & 0001 & 36.5663 & -4.8823 & 4 & 39 & & & $\overline{\mathrm{N}}$ \\
\hline & 0002 & 36.9197 & -4.9233 & 3 & 40 & $\mathrm{M}$ & & $\mathrm{N}$ \\
\hline & 0003 & 36.0714 & -4.2046 & 4 & (35) & $\mathrm{M}$ & & $\mathrm{N}$ \\
\hline & 0004 & 36.9054 & -4.1090 & 4 & 41 & & & $\mathrm{~N}$ \\
\hline \multirow[t]{6}{*}{$1.15-1.25(7662)$} & 0001 & 36.5183 & -4.8925 & 4 & (39) & & & $\overline{\mathrm{N}}$ \\
\hline & 0002 & 36.9264 & -4.9257 & 4 & (40) & $\mathrm{M}$ & & $\mathrm{N}$ \\
\hline & 0003 & 36.0635 & -4.2135 & 4 & (35) & $\mathrm{M}$ & & $\mathrm{N}$ \\
\hline & 0004 & 36.9042 & -4.4438 & 3 & 42 & & & Y \\
\hline & 0005 & 36.8769 & -4.6289 & 4 & 43 & $\mathrm{M} / \mathrm{G}$ & & $\mathrm{Y}$ \\
\hline & 0006 & 36.0565 & -4.0944 & 3 & 44 & & & $\mathrm{~N}$ \\
\hline
\end{tabular}

points (these numbers are representative of numbers of galaxies in real redshift slices as shown in Tables 3 to 5), and we analyse these slices in the same way as real ones. We compute the number of detections depending on the detection thresholds used.

As we proceed in narrow slices, the effect of distant clustering that diminishes the contrast of real structures is also strongly diminished just as well as the bootstrapping does, so mean random fields represent fairly well the actual slice background.

Table 1 shows the numbers of detections in a given slice (whatever the redshift) with respect to the threshold defined in terms of 3 and $4 \sigma$ of the background. This table then estimates the level of wrong structure detections for a given slice. This level remains modest, since there is at most 2 in a given slice at the $3 \sigma$ level and 1 at the $4 \sigma$ level.

\subsection{Global detection rate assessment with Galics simulations and other optical detection methods}

As a first step we generate, using the 50 available GalICS simulations (with $H_{0}=67 \mathrm{~km} \mathrm{~s}^{-1} \mathrm{Mpc}^{-1}, \Omega_{\Lambda}=0.67$ and $\Omega_{\mathrm{m}}=0.33$ ), slices with the same widths as the ones with real data; e.g. Meneux et al. or Blaizot et al. (2006) for a discussion of the ability of these simulations to represent the real universe. These simulations are representative of the general clustering in the whole Universe with the same depth as our sample. For each GalICS slice, we produce mean bootstrapped maps in the same conditions as for real data. In the present stage (see below), we will simply use these simulations to check that the used thresholds lead to a number of detections (see Table 2) that is not totally at odds with the one provided by the real fields. Of course, as 
Table 6. Main structures detected (with available VVDS spectroscopic data).

\begin{tabular}{ccccccccc}
\hline \hline Slice & ID & $\alpha$ & $\delta$ & Gen. ID & N-slice & N-St & $z$ central & $\sigma_{v}\left(\mathrm{~km} \mathrm{~s}^{-1}\right)$ \\
\hline $0.10-0.20$ & 0001 & 36.3789 & -4.2424 & 1 & 3 & 2 & 0.138 & \\
& 0002 & 36.7981 & -4.1970 & 2 & 3 & 2 & 0.185 & \\
\hline $0.15-0.25$ & 0001 & 36.3746 & -4.6831 & 3 & 4 & 2 & 0.225 & \\
& 0003 & 36.6240 & -4.2523 & 5 & 11 & 5 & 0.210 & 258 \\
\hline $0.25-0.35$ & 0001 & 36.3166 & -4.7515 & 10 & 3 & 3 & 0.311 & \\
& 0004 & 36.8416 & -4.5810 & 6 & 12 & 6 & 0.308 & 391 \\
& 0006 & 36.6104 & -4.5286 & 12 & 30 & 21 & 0.313 & 727 \\
\hline $0.45-0.55$ & 0004 & 36.3842 & -4.2726 & 15 & 2 & 2 & 0.542 & \\
& 0005 & 36.8975 & -4.3768 & 16 & 2 & 1 & 0.53 & \\
& 0006 & 36.8481 & -4.6202 & 17 & 4 & 3 & 0.543 & 594 \\
\hline $0.55-0.65$ & 0003 & 36.4645 & -4.4997 & 21 & 15 & 9 & 0.613 & 864 \\
& 0004 & 36.8646 & -4.5484 & 19 & 10 & 6 & 0.610 & \\
\hline $0.60-0.70$ & 0001 & 36.6686 & -4.5096 & 22 & 15 & $7 / 6$ & $0.634 / 0.687$ & $319 / 601$ \\
$0.70-0.80$ & 0003 & 36.8791 & -4.2025 & 28 & 2 & 2 & 0.784 & \\
$0.75-0.85$ & 0006 & 36.5030 & -4.4714 & 29 & 2 & & & 488 \\
$0.85-0.95$ & 0005 & 36.3925 & -4.4135 & 34 & 10 & 5 & 0.920 & \\
\hline
\end{tabular}

Other columns are: ID, coordinates, general identification, $\mathrm{Nb}$ of redshifts in the slice, $\mathrm{Nb}$ of redshifts in the system (when two systems are visible, we give both values), central redshift, velocity dispersion (when more than 4 available redshifts). We restrict here to VVDS redshift selected in the ellipse corresponding to every structure. When no value is given for the mean redshift and velocity dispersion, this means that the sparse sampling and/or the small number of data do not allow a significant characterisation.

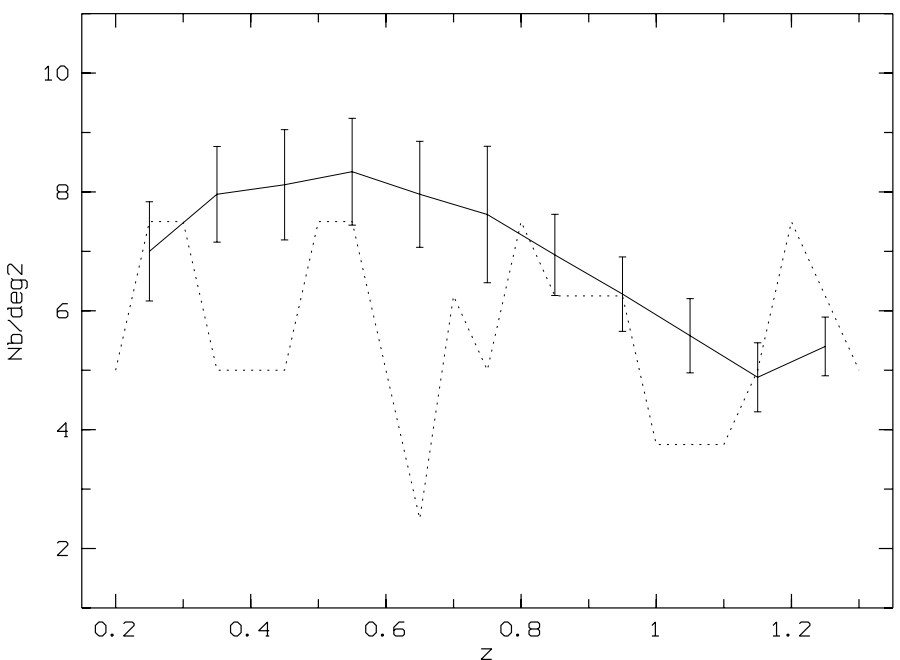

Fig. 4. Number of $3 \sigma$ detections per $\operatorname{deg}^{2}$ in the GalICS simulations: continuous line with error bars. Number of detections per $\mathrm{deg}^{2}$ in the D1 field: dashed line.

well in real data as in simulated ones, false detections (due e.g. to projection effects) and cosmic variance affect the number of structures. Consequently, numbers are expected to agree only in the mean and we recall that a complete evaluation in terms of richness, mass, and other characteristics will be the subject of another paper.

We, however, compare the number of detections in our sample and in the GalICS simulations from Table 2 and Fig. 4. This will give a global estimate of how closely our detections agree with the cosmological model used in the chosen GalICS simulations. Figure 4 shows that our number of detections does agree with the $\left(\Omega_{\Lambda}=0.67\right.$ and $\left.\Omega_{\mathrm{m}}=0.33\right)$ model given the error bar sizes. Of course these detections could be in both cases either real or false detections. However, using the random fields representative of each slice background, as seen above, the highlevel thresholds show that the false detection rate is expected to be rather low. It would be an unlikely coincidence that in every

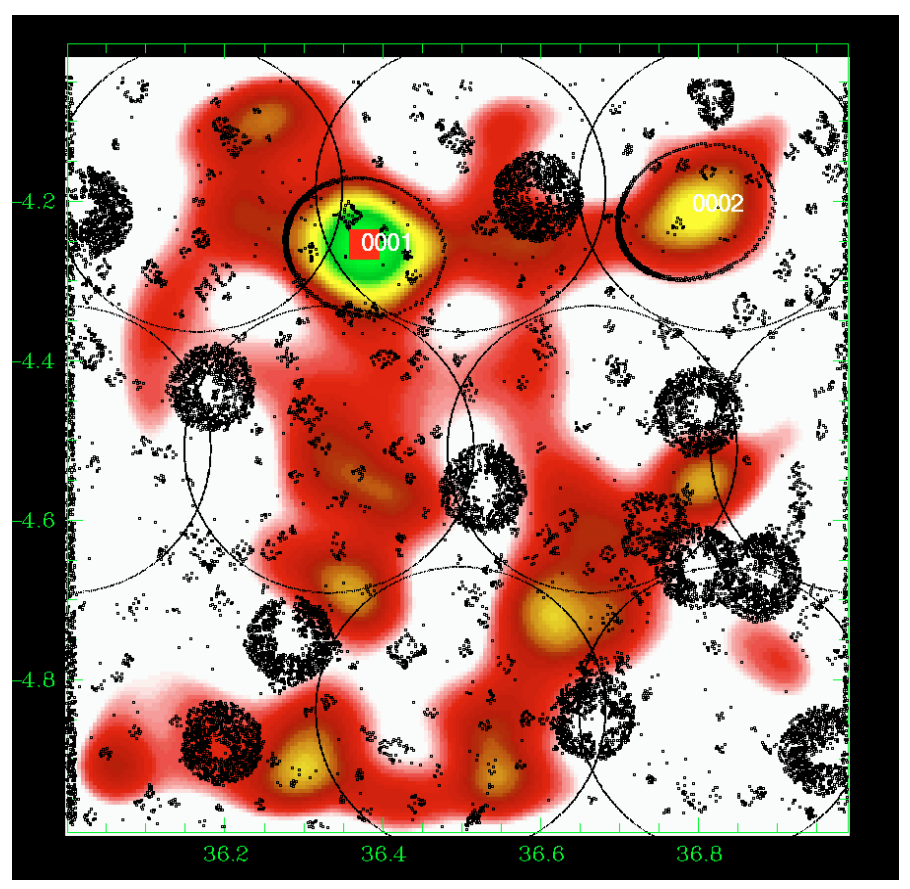

Fig. 5. Slice $0.10-0.20$ (i.e. $0.10 \leq z \leq 0.20$ ). Colour contours are drawn from the mean galaxy density image. Bottom level is the mean value of this density map. Black small dots show the fake galaxies detected in the D1 masked areas. Large circles are the XMM-LSS fields. Peaks are detected at the $3 \sigma$ level (check tables of structures to see which ones are also detected at the $4 \sigma$ level) and can be distinguished by their number. C1 XMM clusters (from Pierre et al. 2006) in the same redshift range (with an allowance of 0.01) are superimposed as red squares, C2 and C3 (from Pierre et al. 2006) as white squares. Coordinates are given in decimal degrees (J2000).

slice, the number of real and false detections conspire to give the right numbers.

We detect less structures at intermediate redshifts $(0.55$; 0.75 ) than predicted by the model. But this also corresponds (see below) to a lack of X-ray detections, revealing that it could be 


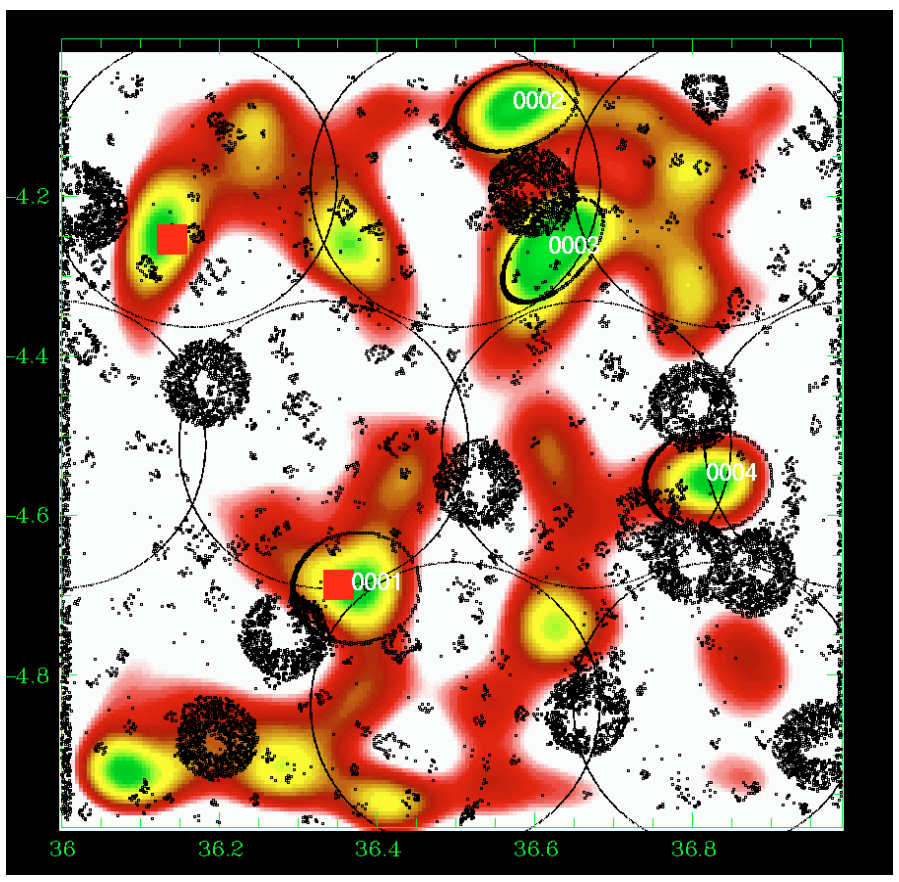

Fig. 6. Same as Fig. 5 for the slice $0.15-0.25$.

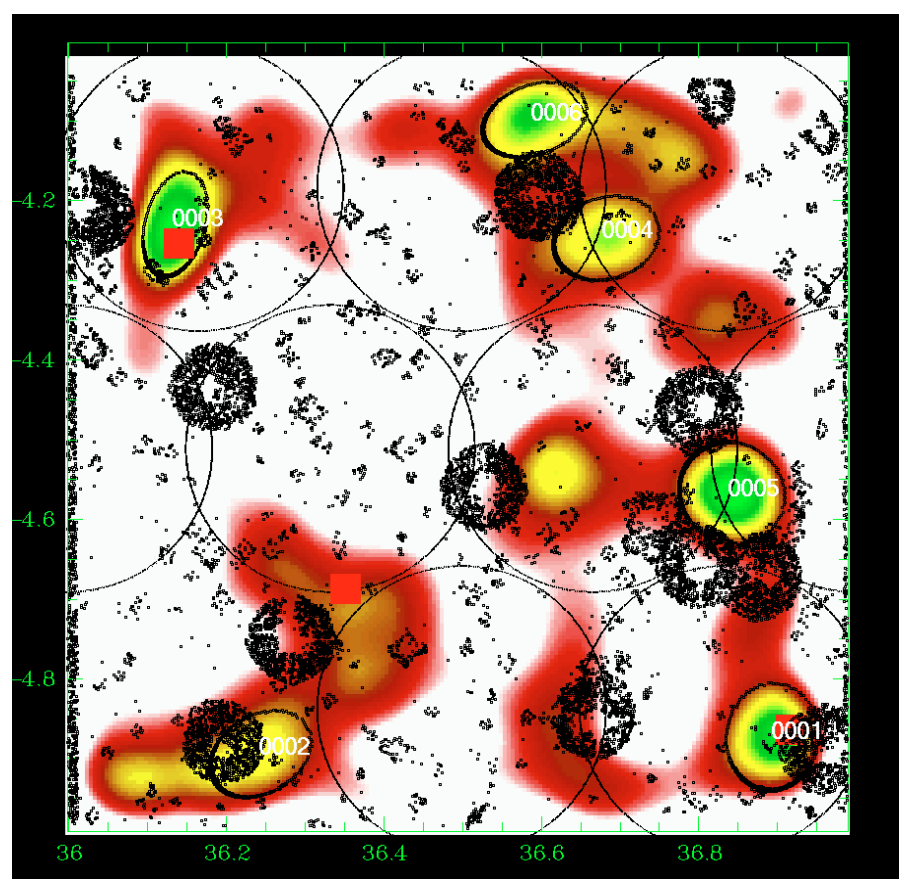

Fig. 7. Same as Fig. 5 for the slice $0.20-0.30$.

an empty region. More precise comparisons are required to constrain the Cosmology, but the relatively good agreement between simulations and observations gives confidence in the method.

As seen from Tables 3 to 5, we detected about 40 independent structures within an area of $0.8 \mathrm{deg}^{2}$ up to $z=1.1$, which is very close to the $52 \pm 8$ per $\mathrm{deg}^{2}$ found by Olsen et al. (2007) using a totally different method and a slightly deeper magnitude limit; but given the considered magnitudes, this should only affect the faint population of our detected structures and not the structures themselves too strongly. A close comparison between the two methods is devoted to another paper.

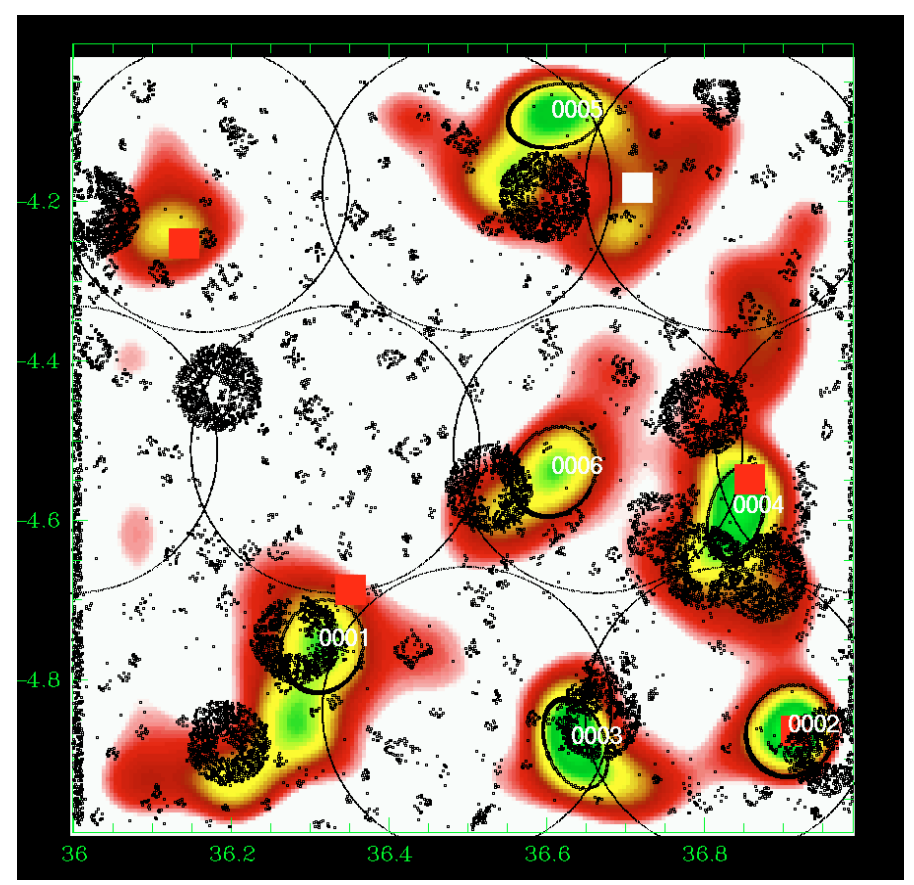

Fig. 8. Same as Fig. 5 for the slice $0.25-0.35$.

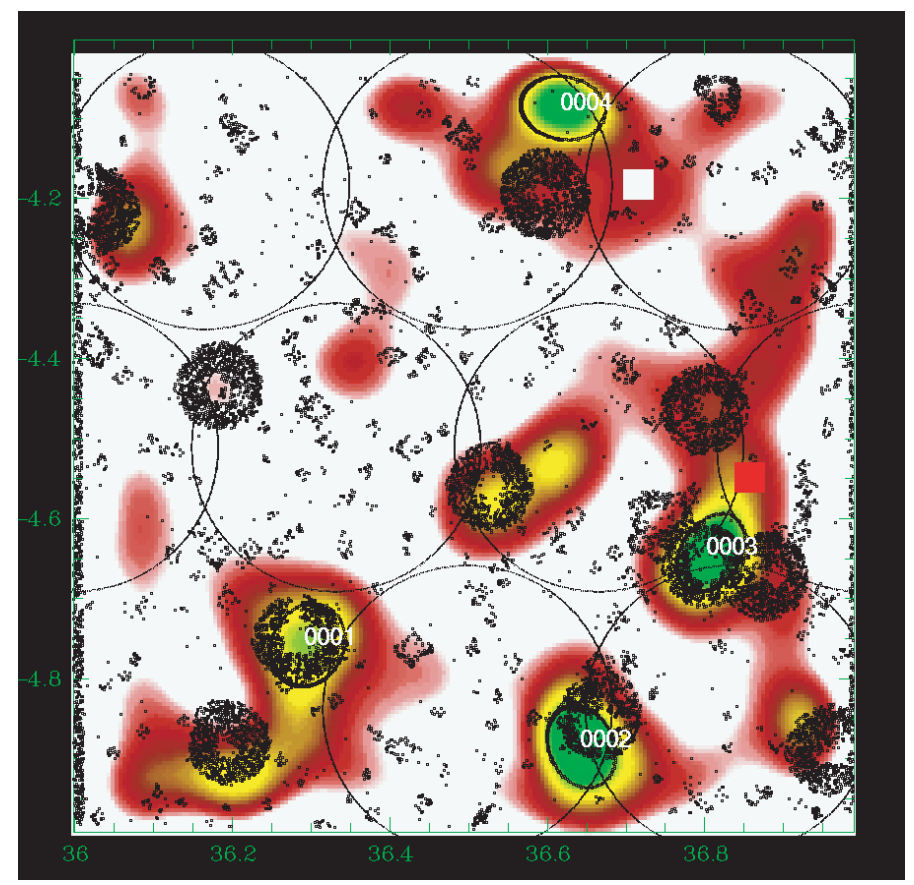

Fig. 9. Same as Fig. 5 for the slice $0.30-0.40$.

\subsection{Comparison with XMM-LSS and the VVDS}

Once false detection problem has been addressed, the next concern is the ability of any method to detect real structures at the right place and at the right distance or instead to explain when and why they are not detected. In other words, what is the socalled detection function?

One way to correctly estimate such a selection function is to use cosmologically simulated catalogues analysed with exactly the same protocol as for the real data. One can also use mock catalogues in which structures are put in by hand allowing the recovery power estimation. However, if it is pedagogical in the sense that it gives a sense of how the algorithm works, it is not 


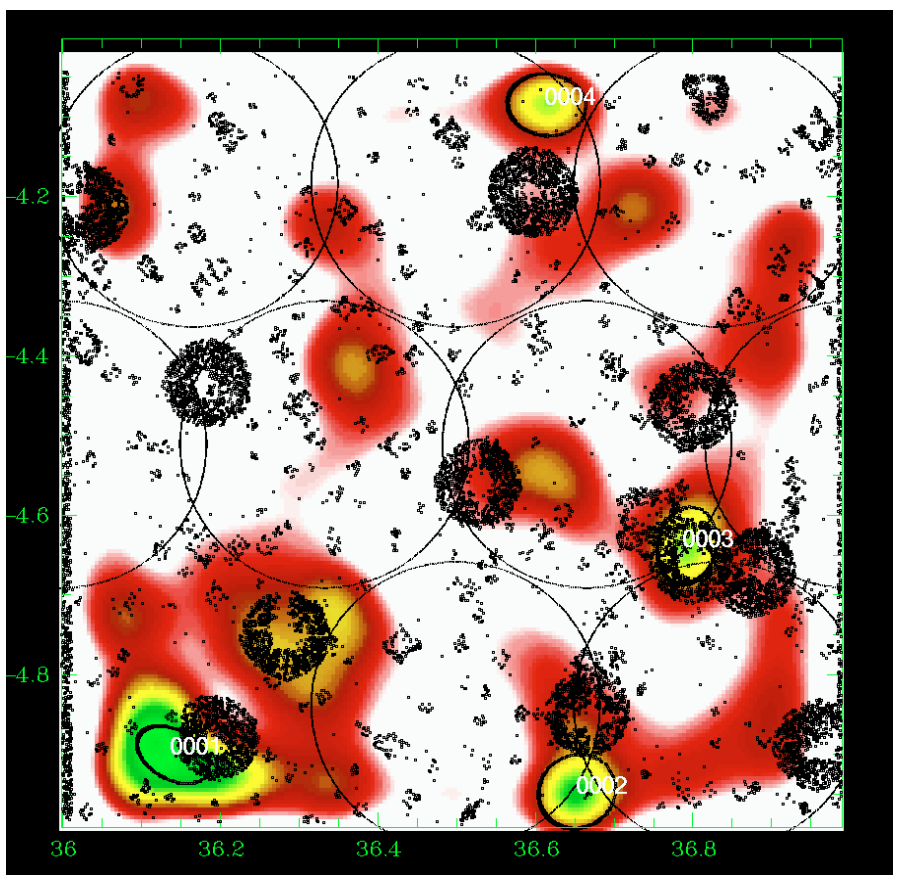

Fig. 10. Same as Fig. 5 for the slice $0.35-0.45$.

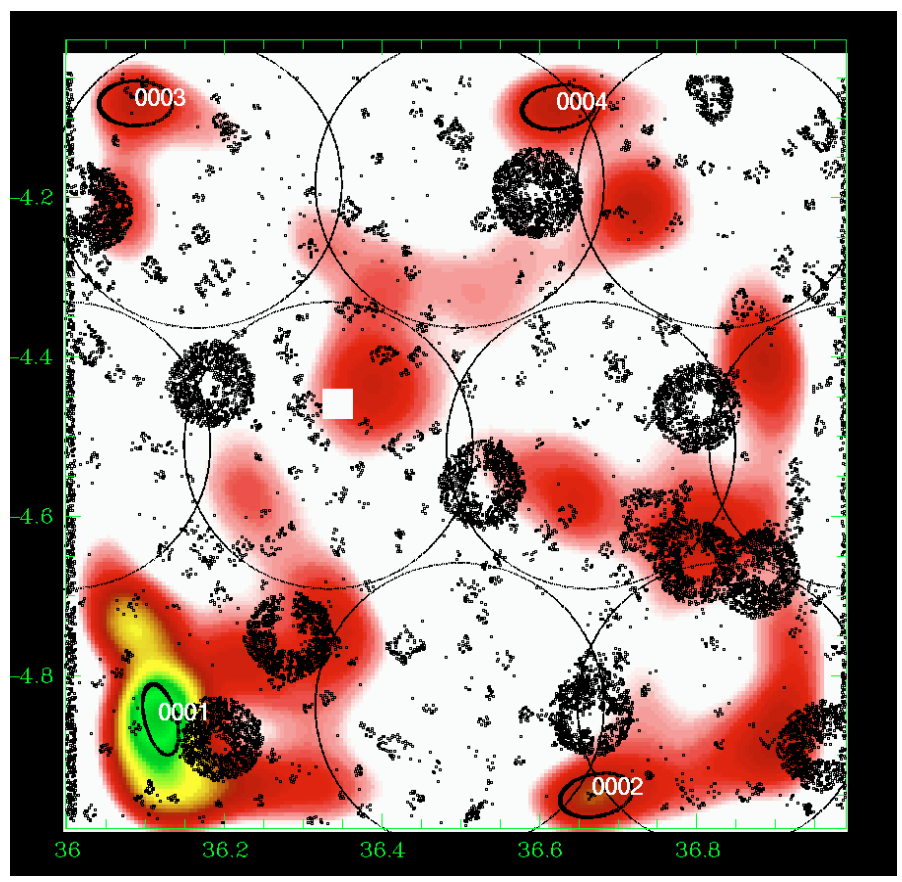

Fig. 11. Same as Fig. 5 for the slice $0.40-0.50$.

always possible to explore all the range of parameters and conditions in a practical way until detailed cosmological simulations adapted to the CFHTLS characteristics are available.

A second way to estimate this selection function is empirical: the use of detections obtained in a totally different way (X-ray emission of groups and clusters detected by the XMM-LSS) accounting in the meantime for spectroscopic information issued from VVDS. This spectroscopic information is used in the following way. For every structure in a given slice, we look at the redshift distribution of the VVDS data along the line of sight, within a $1 \mathrm{Mpc}$ ellipse, up to $z=1.2$ (central redshift of the highest redshift slice considered) and we look for clustering in

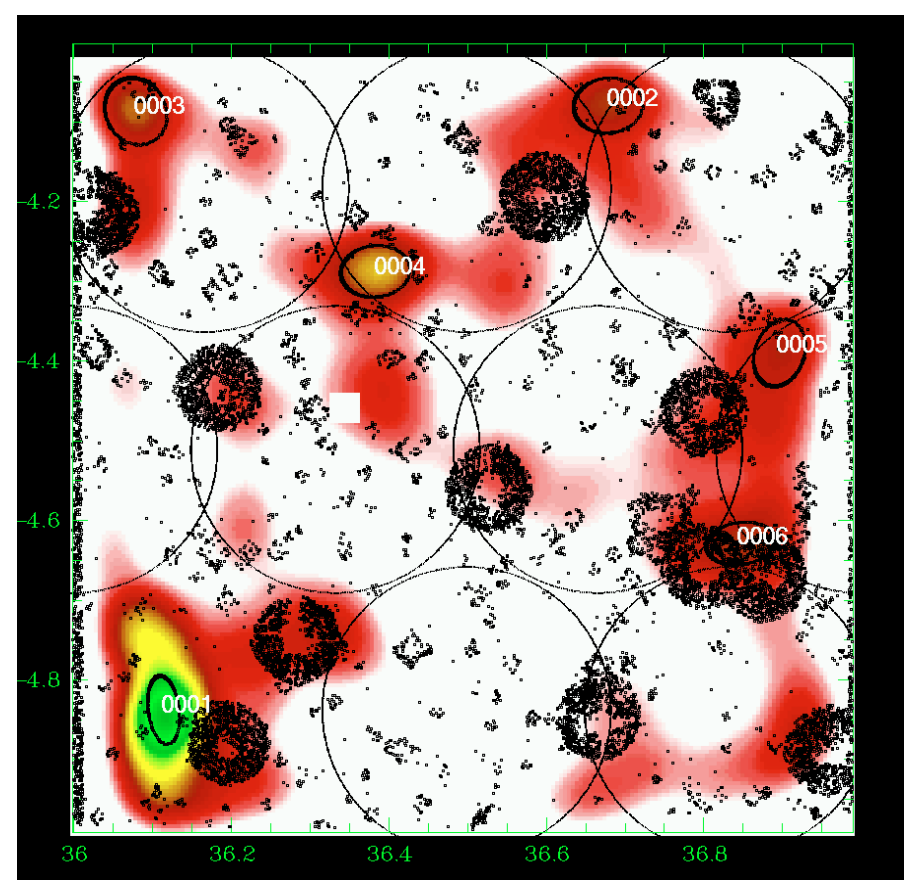

Fig. 12. Same as Fig. 5 for the slice $0.45-0.55$.

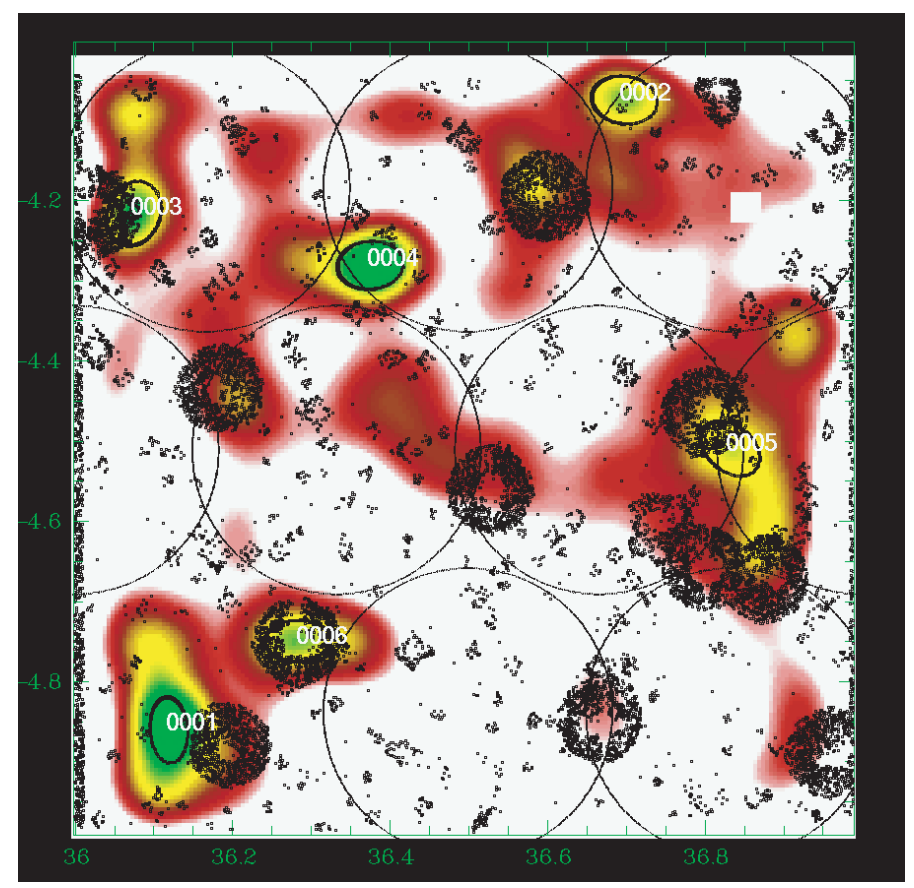

Fig. 13. Same as Fig. 5 for the slice $0.50-0.60$.

spectroscopic redshift within the redshift range of the slice. We put galaxies as possible members of a system when the redshifts of 2 galaxies do not differ by more than $0.0026 \times(1+z)$ (see also Sect. 3.1). All the corresponding numbers of this analysis are given in Table 6.

Here, we check if we recover with the thresholds defined above the various XMM sources or not. Of course, there are physical reasons for not detecting an optical overdensity in X-ray (e.g. if dealing with a non totally virialized system), as well as observational ones since XMM-LSS does not entirely cover D1 and does not have a completely homogeneous exposure time. Conversely, if there is no optical structure when a diffuse X-ray 


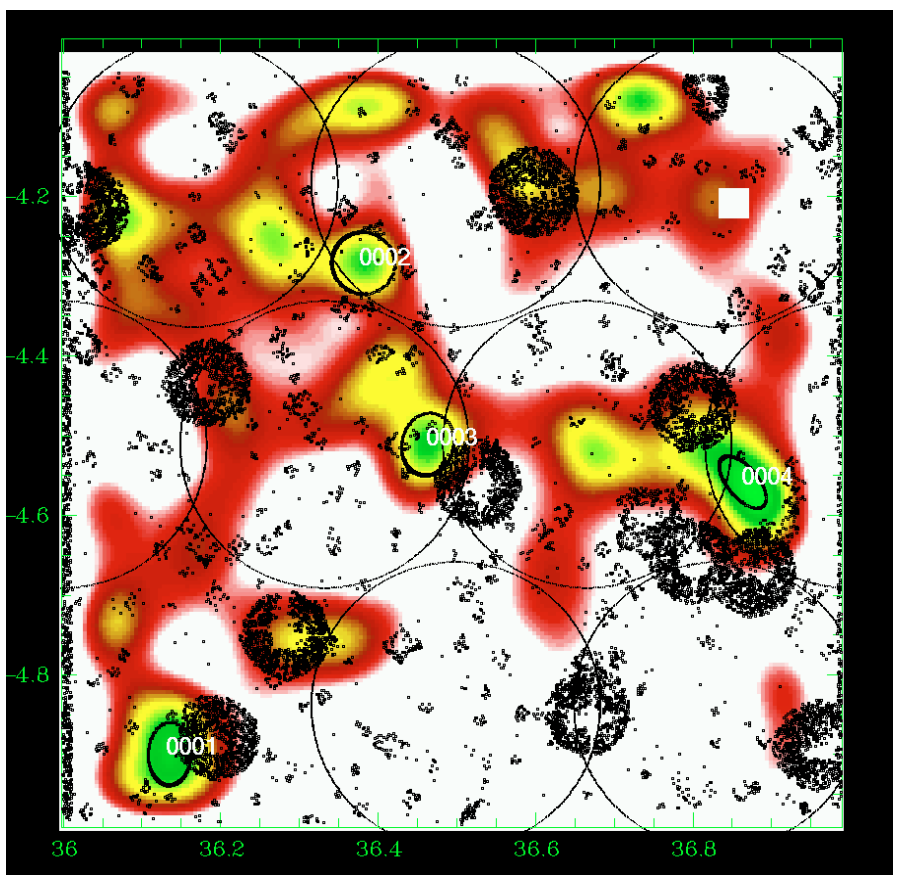

Fig. 14. Same as Fig. 5 for the slice $0.55-0.65$.

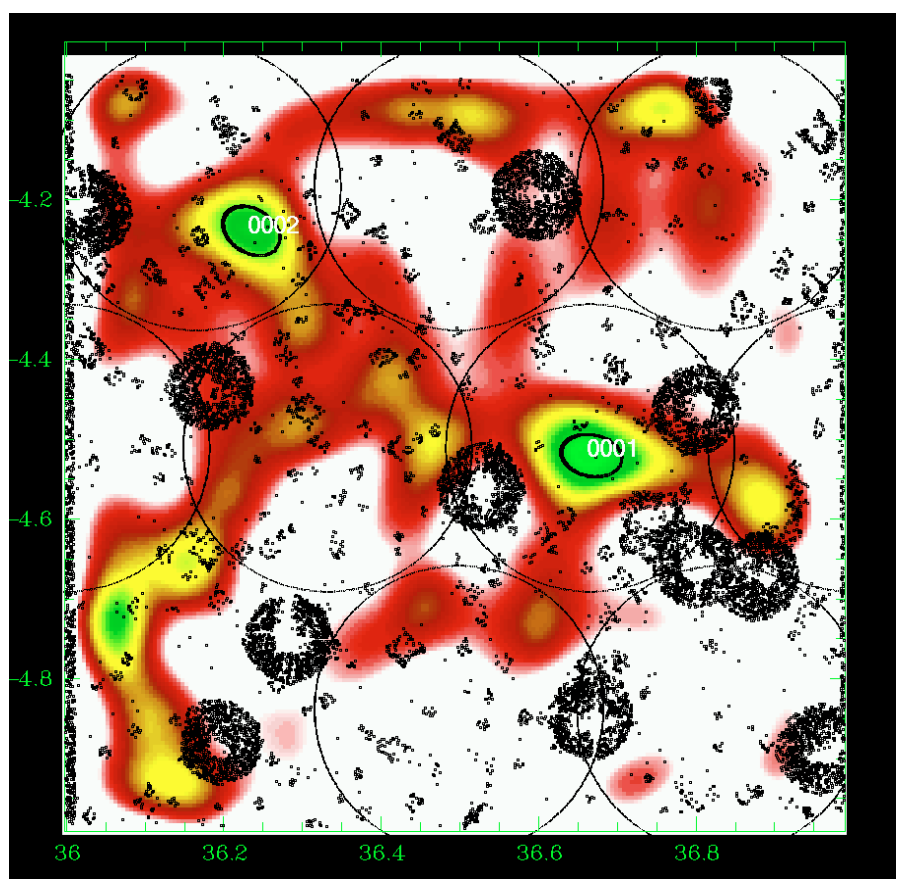

Fig. 15. Same as Fig. 5 for the slice $0.60-0.70$.

source is present, it could reveal a failure of the present method or a mistake in its distance, as well as a peculiar class of extended $\mathrm{X}$-ray structures (as fossil groups that are massive systems with few galaxies, e.g. Jones et al. 2003 or Ulmer et al. 2005).

Figures 5 to 23 show a subsample of the slices up to $z=1.1$ for thresholds of $3 \sigma$. We note that these figures are plotted with the same colour coding, with the lowest level being the mean value of the density map. If different slices exhibit strongly different background noise (or different maximal density values), then significant peaks can appear less prominent in the figures compared to less significant peaks in slices with different noise levels. The individual properties of the structures are given in

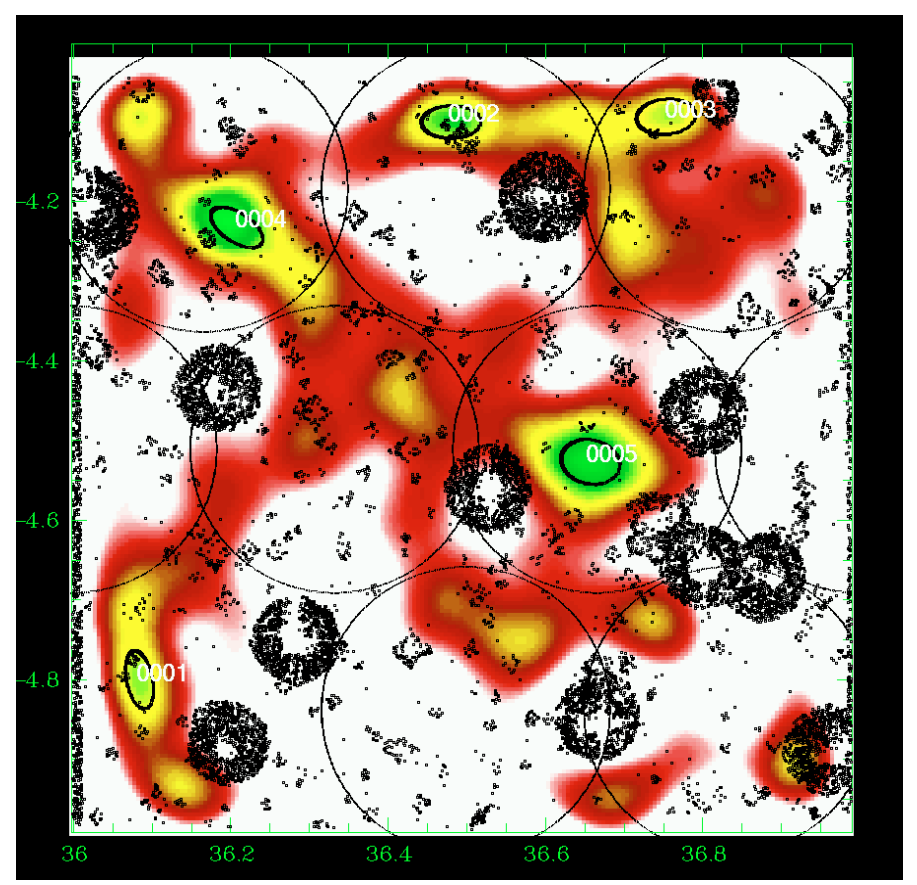

Fig. 16. Same as Fig. 5 for the slice $0.65-0.75$.

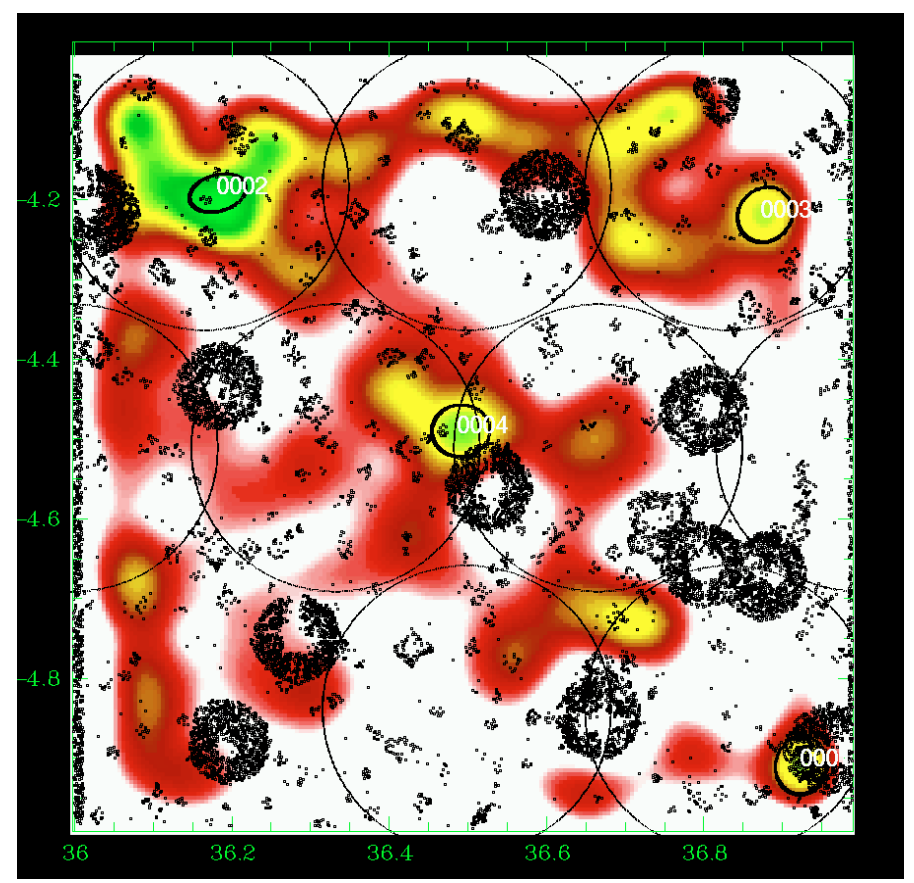

Fig. 17. Same as Fig. 5 for the slice $0.70-0.80$.

Tables 3 to 5 . We note that structure 35 (general identification) is present from $z=0.85$ to $z=1.25$ with a small position shift on the sky. Structure 29 (general identification) is perhaps identified with the C3-c cluster (Pierre et al. 2006), but the case is not closed as it is located very close to a masked region. We overplotted XMM-LSS clusters in the range 0.1-1.2 with the following rule: $\mathrm{C} 1, \mathrm{C} 2$, and C3 XMM-LSS clusters were plotted in our graphs when included in the considered photometric redshift range (with an allowance of 0.01, only useful for C1-025).

In the tables, when a given structure is detected in adjacent slices, we always selected the one corresponding to the highest significance level to identify it with an X-ray source. The 


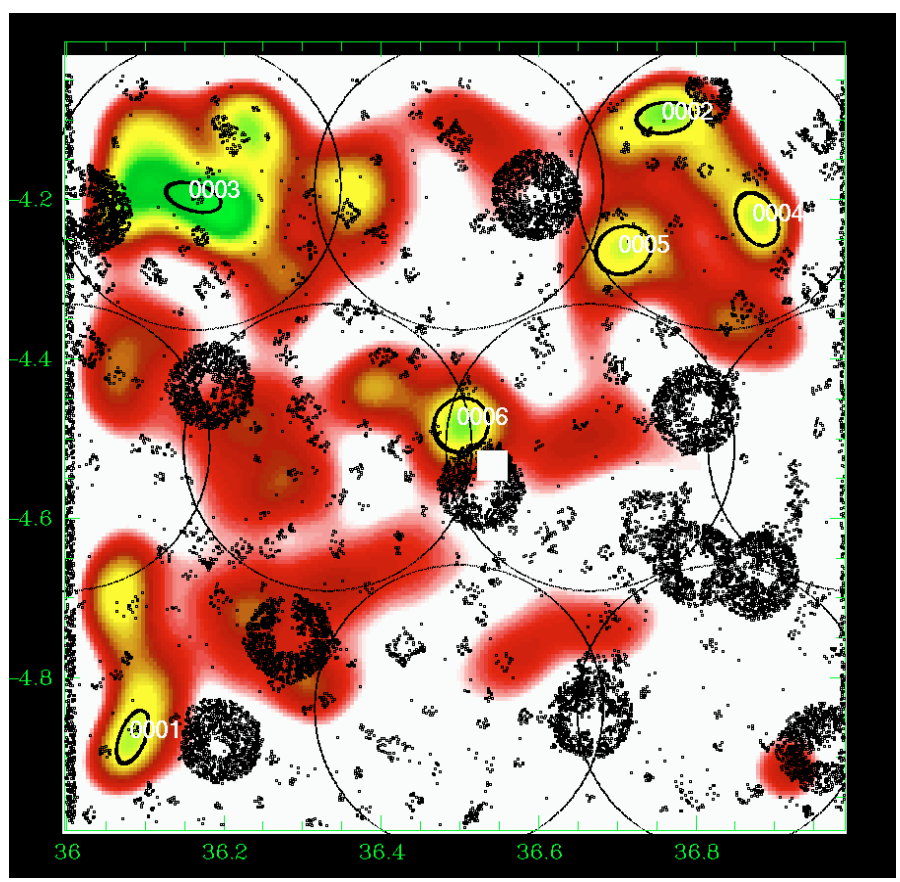

Fig. 18. Same as Fig. 5 for the slice $0.75-0.85$.

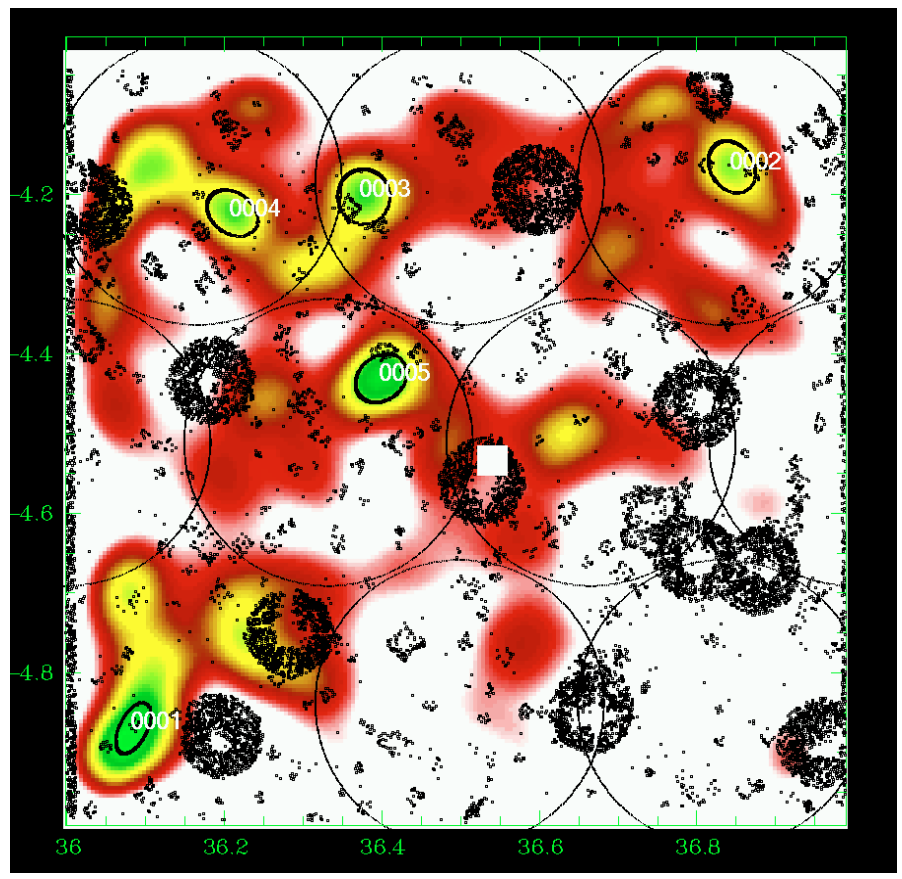

Fig. 19. Same as Fig. 5 for the slice $0.80-0.90$.

X-ray position has to be included in the optical detection ellipse. Finally, in the tables, we also take into account the XMMLSS clusters provided by Andreon et al. (2005) and Willis et al. (2005) in specific analyses, separate from Pierre et al. (2006), but using the same XMM data.

- First, it should be noted that, for redshifts higher than 0.1 , all XMM-LSS C1 clusters are detected at the $3 \sigma$ level with the present method (except for C1-029 almost outside the optical field and in a masked region of this field). Most of these are also detected at the $4 \sigma$ level except C1-005 (detected at the $3.75 \sigma$ level) and C1-025 (at 3.3 $\sigma$ level).

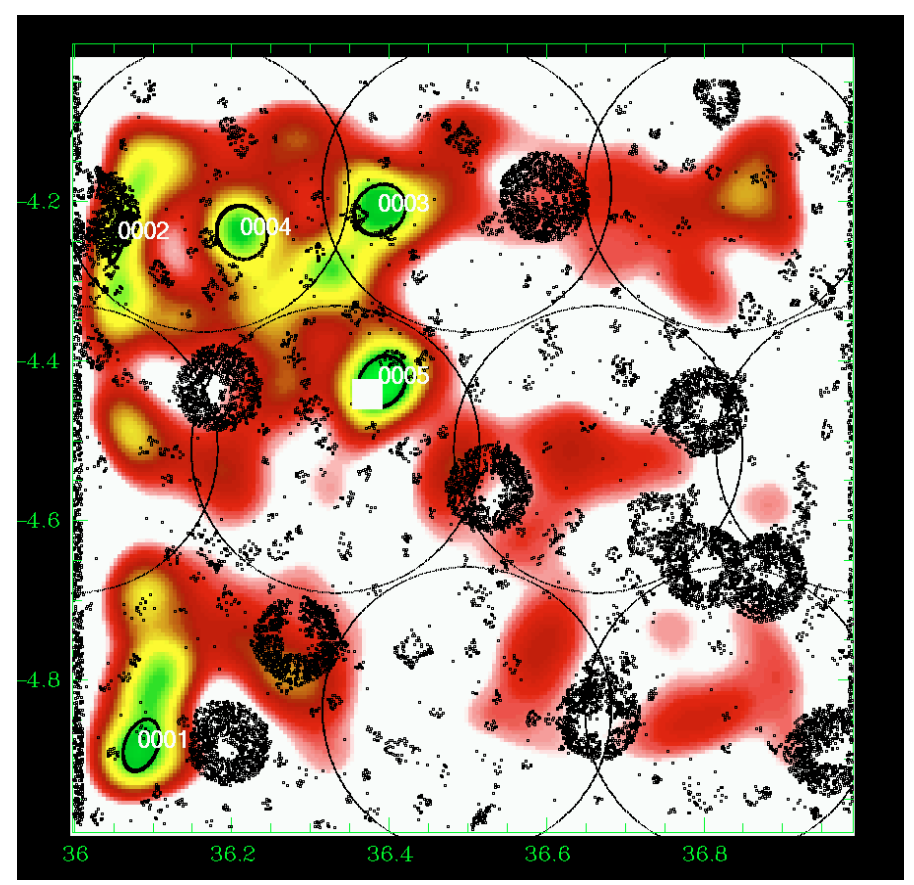

Fig. 20. Same as Fig. 5 for the slice $0.85-0.95$.

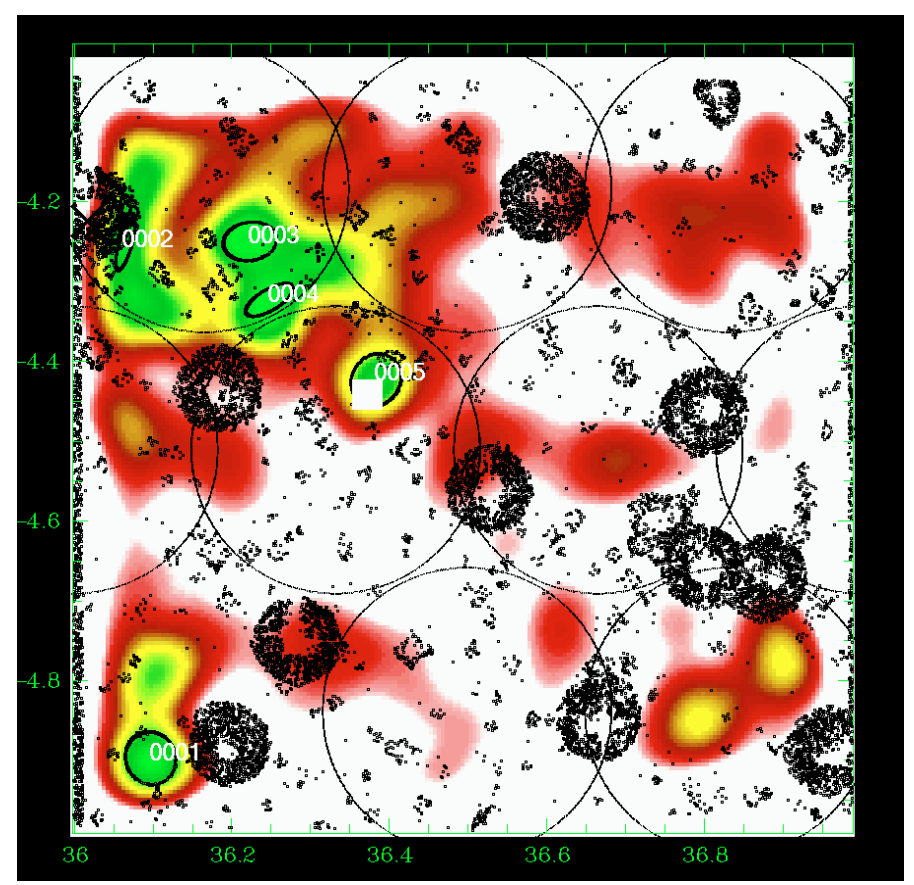

Fig. 21. Same as Fig. 5 for the slice 0.90-1.00.

- C2-038 is not detected in our analysis and is neither in a masked nor in an edge region. Looking in more detail at the maps shows that it is detected at only a $1.5 \sigma$ level in the $[0.50 ; 0.60]$ slice.

- We also detect 2 out of the 4 C3 clusters. Surprisingly, the C3 detected are at high $z$, while the non-detected ones are at low redshift. However, C3-a appears close to a bright star and is probably spoiled in the CFHTLS data (masked region). For C3-d, it also appears to be detected at only $1.5 \sigma$ in the $[0.30$; $0.40]$ slice. 


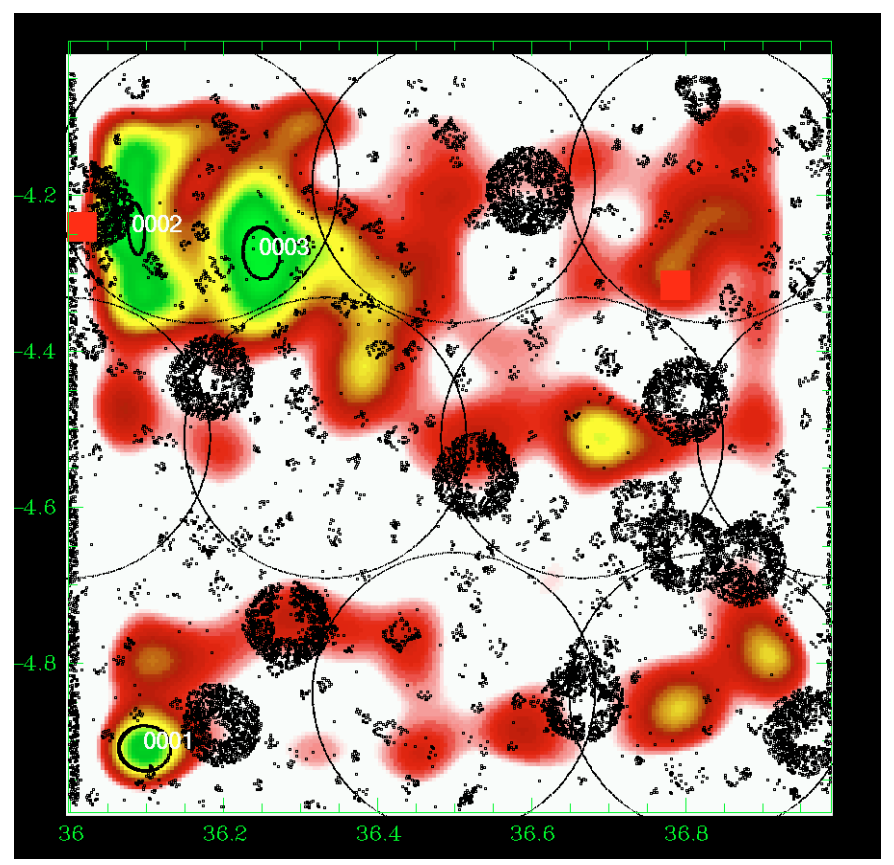

Fig. 22. Same as Fig. 5 for the slice $0.95-1.05$.

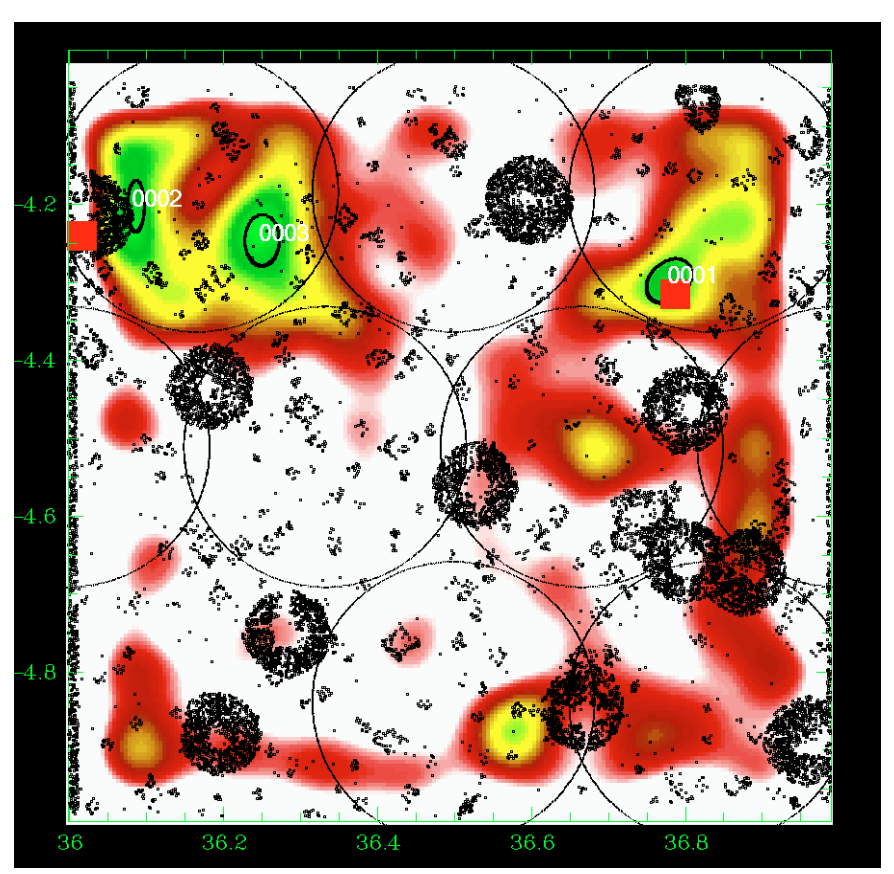

Fig. 23. Same as Fig. 5 for the slice 1.00-1.10.

- We finally find coincidence in the correct redshift range (see Tables 3 to 5) for structures 4 and 34 with the X-ray sources confirmed by Andreon et al. (2005) and Willis et al. (2005).

- It must be underlined that our analysis succeeds in recovering the cluster 0004 (number 15) of the $[0.50 ; 0.60]$ redshift slice afterwards, identified as a faint X-ray source but rejected as a possible extended source from the XMM-LSS list in a first stage (so not included in the $\mathrm{C} 1 / \mathrm{C} 2 / \mathrm{C} 3$ classification). This X-ray source is very close to the bright XMMLSS C1 cluster 041 but is still detected by our method (see Figs. 12 and 26). This illustrates the ability of our method to efficiently disentangle nearly superposed clusters. We also find detections apparently without any X-ray identification.
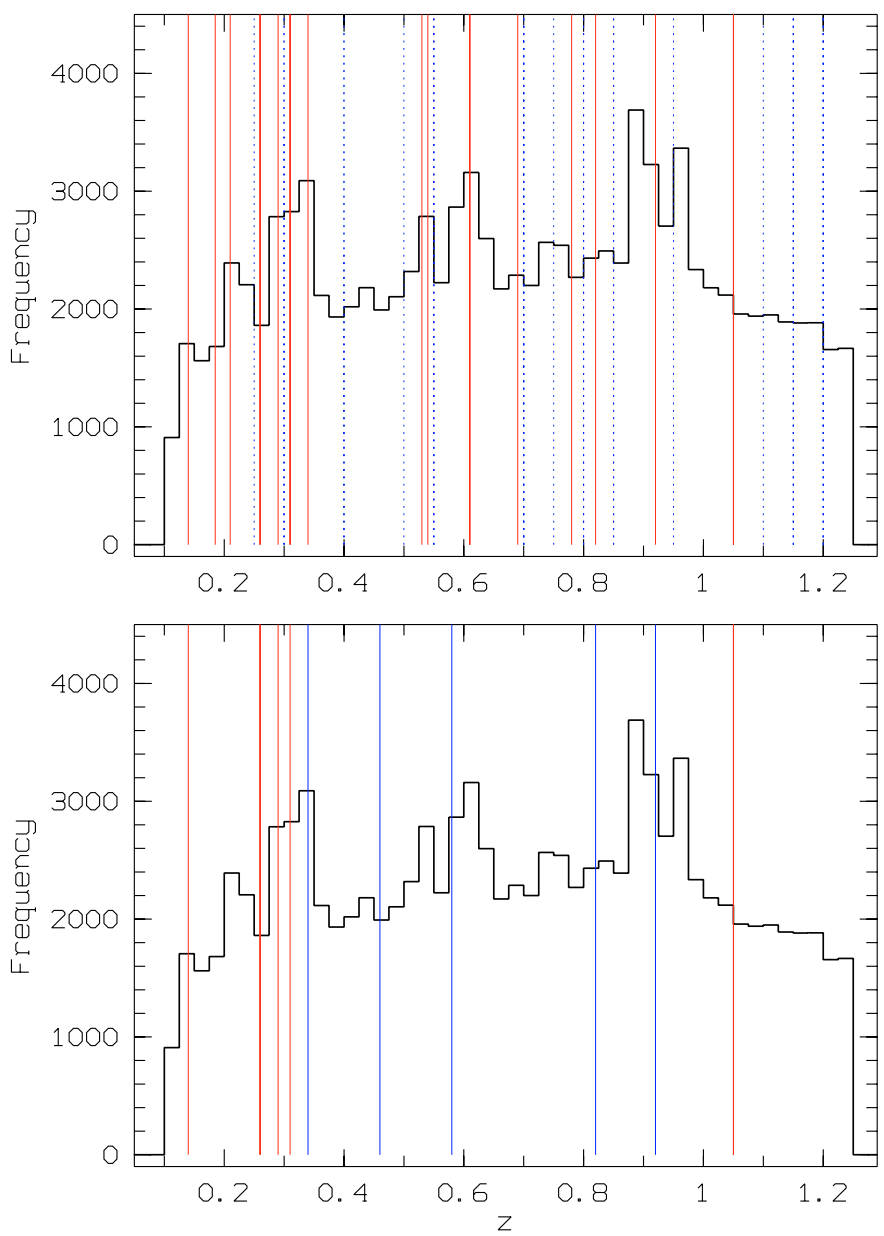

Fig. 24. Histogram of all photometric redshifts along the line of sight between $z=0.1$ and 1.25 overplotted with detected structures. Upper figure: structures detected with the photometric redshifts. Those with a precise redshift determination (first from XMM-LSS papers and second from VVDS spectroscopic data) are the red continuous lines. The blue dashed lines are clusters with only the photometric redshift determination (taken as the central redshift of the considered slice). Lower figure: structures detected in X-rays from Pierre et al. (2006). C1 clusters are the red lines. C2 and C3 clusters are the blue lines.

One clear example is given by structure 12 detected here at the $3 \sigma$ level without confirmed X-ray counterpart in the redshift bin. For this region, however, more than 30 VVDS redshifts are available confirming definite clustering at $z \sim 0.31$.

In summary, there are $1 \mathrm{C} 2$ cluster and $2 \mathrm{C} 3$ clusters that are not detected at the 3- $\sigma$ level (but 2 are recovered at lower levels) with no obvious explanation among the confirmed clusters from Pierre et al. (2006). This corresponds to a level of $\sim 15 \%$ that turns out to be the number of structures our method completely missed compared to X-ray methods.

Conversely, several structures are detected in the visible at a significant level with no counterparts in X-ray: are they real or false detections? Restricting ourselves to areas in common, with no biases (i.e. excluding A, G, E, M, S regions: see Tables 3 to 5 ), and limiting ourselves to $z \leq 1.05$ (the limit in redshift of Pierre et al. 2006), we find 11 such structures of which 5 have spectroscopic information. Namely these are systems $2,16,19$, 21, and 28 (see Table 6) and are therefore likely to be real. We also note that structure number 3 (general ID) is detected at $z=$ 0.26 by Pierre et al. (2006) and at $z=0.225$ using only VVDS redshifts. This discrepancy is probably due to the small number 

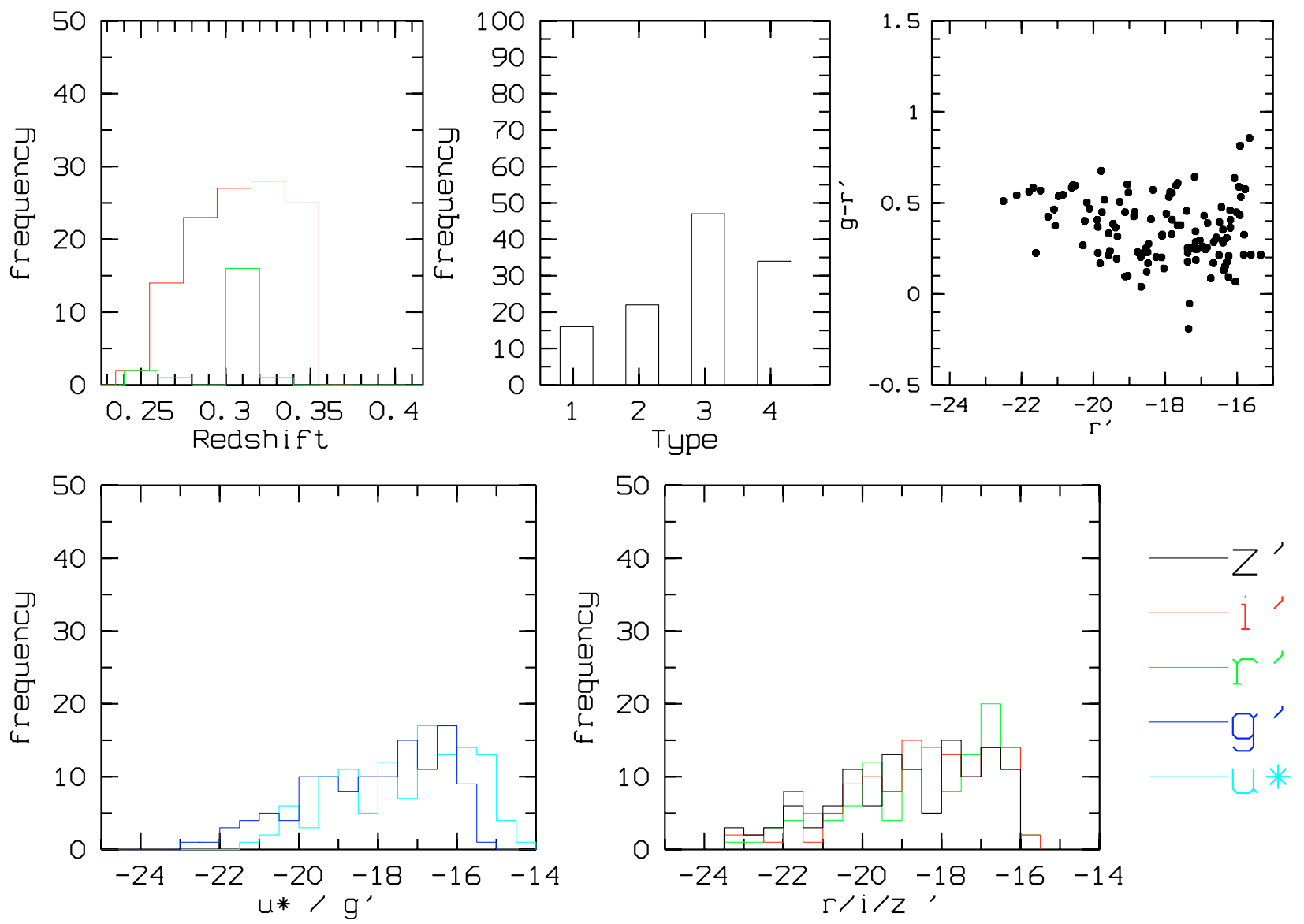

Fig. 25. Summary of the properties for the candidate 0004 in the [0.25; 0.35] redshift slice. Lower left: $u^{*}$ and $g^{\prime}$ band absolute magnitude histograms; lower right: $r^{\prime}, i^{\prime}, z^{\prime}$ absolute magnitude histograms; upper left: redshift histograms (photometric redshifts: red, spectroscopic redshifts: green); upper centre: spectrophotometric types (following Coleman et al. 1980); upper right: colour-magnitude relation.

of VVDS redshifts (4) in the considered slice, leading to a wrong estimate.

We conclude that we are probably more efficient at detecting very low-mass and galaxy-dominated systems (as compared to gas or dark-matter dominated systems) than are X-ray methods. These 11 , only optically-detected, structures is the number of structures probably missed by the X-ray method.

\subsection{Clusters, groups, and filament properties}

A future paper will be dedicated to precise study of the properties of these structures, but we show in Fig. 24 the histogram of all photometric redshifts along the line of sight between $z=0.1$ and 1.25 overplotted with detected structures. We clearly see that we can detect structures in almost all galaxy concentrations in the redshift space. We also detect several structures in lowdensity regions.

We also present an example of what can be done. Figure 25 shows for example the luminosity functions in the CFHTLS $u^{*}$, $g^{\prime}, r^{\prime}, i^{\prime}$, and $z^{\prime}$ bands of the candidate 0004 in the $[0.25 ; 0.35]$ redshift slice. Objects are selected in the slice (so we still have some foreground and background contamination by galaxies in the considered slice thar are not physically included in the structures) and within the $1 \mathrm{Mpc}$ ellipse. It also shows the redshift and spectro-morphological type histograms (following Coleman et al. 1980), as well as the red sequence in the colour-magnitude relation.
Another remark concerns the detection of structures in several redshift slices. Two such structures (limiting ourselves to those not heavily polluted by CFHTLS masking candidates and to $z$ lower than 1.05: structures 4 and 23) extend over redshift intervals of strictly more than 0.3 and are detected in each of the successive bins at the 3- $\sigma$ level (see Table 7). This interval of 0.3 represents \pm 3 times the typical photometric redshift uncertainty. It is also also larger than the catastrophic errors. This ensures that we are probably not dealing with artefacts. It can still be chance alignements of real structures. However, if not, this is really a puzzling fact, as the length of these filaments (or structure chains) is several hundred Mpc! Their radial extension is clearly larger than the maximal void sizes computed in Hoyle \& Vogeley (2004). If these filaments are real, then they have to cross at least one node (the place where the massive clusters form) of the cosmic web and to percolate from a cosmic cell to another one. We should therefore detect massive clusters inside these filaments in the XMM-LSS data, and these are associated with X-ray structures.

\section{Conclusions}

We show in this paper that using the excellent quality photometric redshifts computed on the D1 CFHTLS field by Ilbert et al. (2006) and combining them with an adaptative kernel galaxy density estimate, we were able to efficiently detect structures up to $z \sim 1.05$ without any hypotheses on the nature of the structures. The analysis based on slices in redshift space allows to 


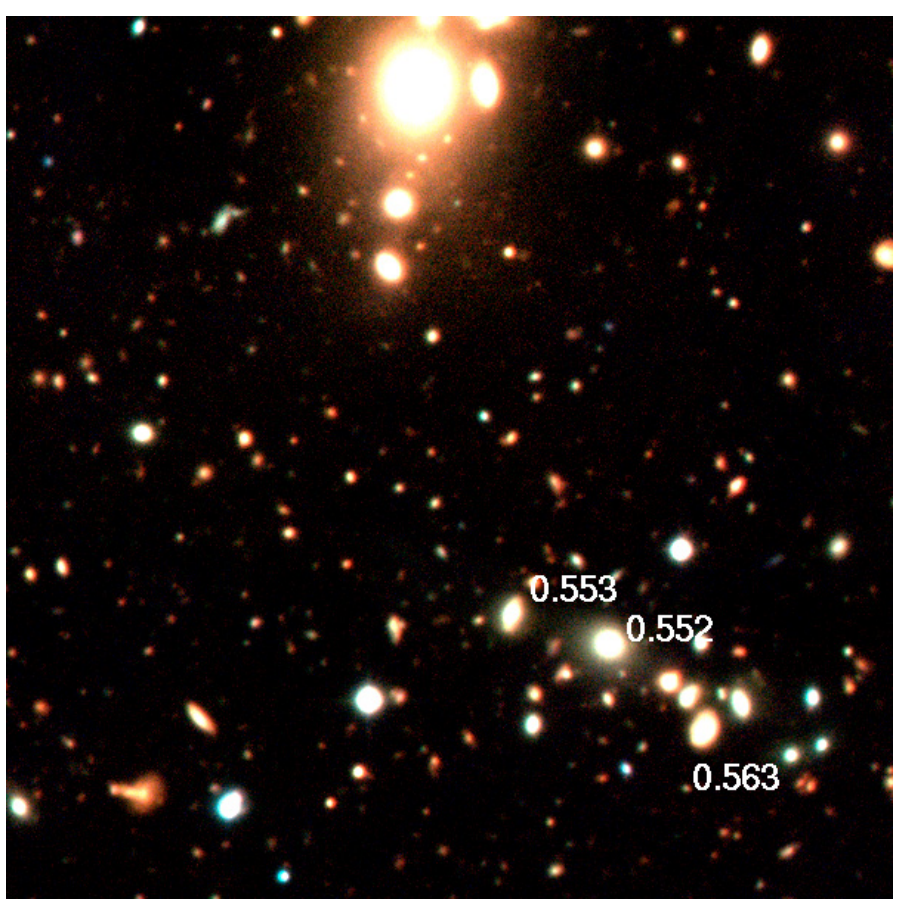

Fig. 26. Trichromic $r / i / z$ CFHTLS image of candidate 0004 in the slice 0.50-0.60. The three XMM-LSS spectroscopic redshifts (distinct from the 2 VVDS redshifts) are shown. The large galaxy at the top of the image is related to the XMM-LSS C1 cluster 041 at $z=0.14$.

Table 7. Redshift detection interval of the radial filaments, mean coordinates, X-ray association, redshift extension in $\mathrm{Mpc}$, and redshift extension in Mpc quadratically diminished with the $\pm 1 \sigma$ photometric redshift uncertainty at the given redshift.

\begin{tabular}{cccccc}
\hline \hline$z$ det. int. & $\alpha$ & $\delta$ & X-ray & Red. ext. & Corr. ext. \\
\hline $1.15-0.60$ & 36.25 & -4.20 & yes & $1467 \mathrm{Mpc}$ & $1421 \mathrm{Mpc}$ \\
$0.60-0.15$ & 36.60 & -4.05 & yes & $1632 \mathrm{Mpc}$ & $1581 \mathrm{Mpc}$ \\
\hline
\end{tabular}

reduce efficiently fore and background contamination, then increasing the contrast of real structures.

Our detections, taking biases of both analyses into account, are in good agreement with X-ray detections (and sometimes help to recover them) and also allowed low mass structures, invisible for X-ray surveys, to be detected. Detections with no evident X-ray counterpart are in general confirmed by spectroscopic information when available. The efficiency of the method also seems to be due to the fact that light appears to trace mass in clusters, which has been verified at least for low redshifts (e.g. Katgert et al. 2004). It is then encouraging to use our method in parallel with others to count clusters both in simulations with realistic galaxy representation and in the real universe. We detected at least two structure-chains of several hundreds of $\mathrm{Mpc}$ (structures 4 and 23). The size of the D1 field is, however, far too small to conduce quantitative cosmological studies, but it allowed calibration of our method. Such quantitative studies will be achieved in future works using other large-scale and deep CFHTLS fields.

Acknowledgements. The authors thank C. Benoist and L.F. Olsen for useful discussions. This work is based in part on data obtained with the European Southern Observatory in Paranal, Chile, and on observations obtained with MegaPrime/Megacam, a joint project of the CFHT and CEA/DAPNIA, at the Canada France Hawaii Telescope (CFHT), which is operated by the National Research Council (NRC) of Canada, the Institut National des Science de l'Univers of the Centre National de la Recherche Scientifique (CNRS) of France, and the University of Hawaii. This work is based in part on data products produced at TERAPIX and the Canadian Astronomy Data Centre as part of the Canada-France-Hawaii Telescope Legacy Survey, a collaborative project of NRC and CNRS. This work is based in part on observations obtained with XMM-Newton, an ESA science mission with instruments and contributions directly funded by ESA Member states and NASA. The VLT-VIMOS observations were carried out on guaranteed time (GTO) allocated by the European Southern Observatory (ESO) to the VIRMOS consortium, under a contractual agreement between the Centre National de la Recherche Scientifique of France, heading a consortium of French and Italian institutes, and ESO, to design, manufacture, and test the VIMOS instrument.

\section{References}

Adami, C., Mazure, A., Ilbert, O., et al. 2005, A\&A, 443, 805 Andreon, S., Valtchanov, I., Jones, L. R., et al. 2005, MNRAS, 359, 1250 Beers, T. C., Gebhardt, K., Forman, W., Huchra, J. P., \& Jones, C. 1991, AJ, 102, 1581

Bertin, E., \& Arnouts, S. 1996, A\&AS, 124, 163

Biviano, A., Durret, F., Gerbal, D., et al. 1996, A\&A, 311, 95

Blaizot, J., Szapudi, I., Colombi, S., et al. 2006, MNRAS, 369, 1009

Coleman, G. D., Wu, C. C., \& Weedman, D. W. 1980, ApJS, 43, 393

Cucciati, O., Iovino, A., Marinoni, C., et al. 2006, A\&A, 458, 39

Dressler, A., \& Shectman, S. A. 1988, AJ, 95, 985

Escalera, E., \& Mazure, A. 1992, ApJ, 388, 23

Forbes, D. A., Ponman, T., Pearce, F., et al. 2006, PASA, 23, 38

Gavazzi, R., \& Soucail, G. 2006, [arXiv: astro-ph/0605591]

Gladders, M. D., \& Yee, H. K. C. 2000, AJ, 120, 2148

Gladders, M. D., \& Yee, H. K. C. 2005, ApJS, 157, 1

Hamana, T., Miyazaki, S., Shimasaku, K., et al. 2003, ApJ, 597, 98

Hoyle, F., \& Vogeley, M. S. 2004, ApJ, 607, 751

Ilbert, O., Arnouts S., McCracken, H. J., et al. 2006, A\&A, 457, 841

Jones, L. R., Ponman, T. J., Horton, A., et al. 2003, MNRAS, 343, 627

Katgert, P., Biviano, A., \& Mazure, A. 2004, ApJ, 600, 657

Le Fèvre, O., Vettolani, G., Garilli, B., et al. 2005, A\&A, 439, 845

Meneux, B., Le Fèvre, O., Guzzo, L., et al. 2006, A\&A, 452, 387

Miller, C. J., Nichol, R. C., Reichart, D., et al. 2005, AJ, 130, 968

Olsen, L. F., Benoist, C., Cappi A., et al. 2007, A\&A, 461, 81

Postman, M., Lubin, L. M., Gunn, J. E., et al. 1996, AJ, 111, 615

Pierre, M., Pacaud F., Duc, P.-A., et al. 2006, MNRAS, 372, 591

Slezak, E., Durret, F., \& Gerbal, D. 1994, AJ, 108, 1996

Tang, J. Y., \& Fan, Z. H. 2005 ApJ, 635, 60

Ulmer, M. P., Adami, C., Covone, G., et al. 2005, ApJ, 624, 124

Willis, J. P., Pacaud, F., Valtchanov, I., et al. 2005, MNRAS, 363, 675 
A. Mazure et al.: Structure detection in the D1 CFHTLS deep field using accurate photometric redshifts: a benchmark, Online Material $p$ I

\section{Online Material}


A. Mazure et al.: Structure detection in the D1 CFHTLS deep field using accurate photometric redshifts: a benchmark, Online Material $p 2$

Table A.1. Same as Tables 3 to 5 for slices with central redshifts between 1.25 to 1.425.

\begin{tabular}{|c|c|c|c|c|c|c|c|c|c|}
\hline Slice & Structure ID & $\alpha$ & $\delta$ & Threshold in $\sigma$ & Gen. ID & X-ray ID and $z$ XMM & $\overline{T-X}$ & $\overline{\text { VVDS }}$ & $\overline{\mathrm{Nb}}$ \\
\hline \multirow{5}{*}{$1.20-1.30(7174)$} & 0001 & 36.9239 & -4.9189 & 4 & $(40)$ & $\mathrm{M}$ & & $\mathrm{N}$ & \\
\hline & 0002 & 36.4878 & -4.9125 & 4 & (39) & & & $\mathrm{N}$ & \\
\hline & 0003 & 36.9052 & -4.4377 & 4 & (42) & & & Y & 1 \\
\hline & 0004 & 36.8669 & -4.6201 & 4 & (43) & $\mathrm{M} / \mathrm{G}$ & & $\mathrm{N}$ & \\
\hline & 0005 & 36.0596 & -4.0896 & 3 & (44) & & & $\mathrm{N}$ & \\
\hline \multirow[t]{4}{*}{$1.25-1.35(6352)$} & 0001 & 36.7340 & -4.7891 & 4 & 45 & & & $\mathrm{~N}$ & \\
\hline & 0002 & 36.0588 & -4.1025 & 3 & (44) & & & $\mathrm{N}$ & \\
\hline & 0003 & 36.8989 & -4.4477 & 4 & (42) & & & $\mathrm{N}$ & \\
\hline & 0004 & 36.8744 & -4.6244 & 3 & (43) & $\mathrm{M} / \mathrm{G}$ & & $\mathrm{N}$ & \\
\hline \multirow[t]{2}{*}{$1.30-1.40(5004)$} & 0001 & 36.7386 & -4.7950 & 4 & (45) & & & $\bar{N}$ & \\
\hline & 0002 & 36.8913 & -4.4610 & 3 & (42) & & & $\mathrm{Y}$ & 1 \\
\hline \multirow[t]{3}{*}{$1.35-1.50(5673)$} & 0001 & 36.8357 & -4.6087 & 3 & 46 & $\mathrm{M} / \mathrm{G}$ & & $\mathrm{N}$ & \\
\hline & 0002 & 36.3528 & -4.1316 & 3 & 47 & $\mathrm{~S}$ & & $\mathrm{~N}$ & \\
\hline & 0003 & 36.9115 & -4.4723 & 3 & $(42)$ & & & $\mathrm{N}$ & \\
\hline
\end{tabular}

